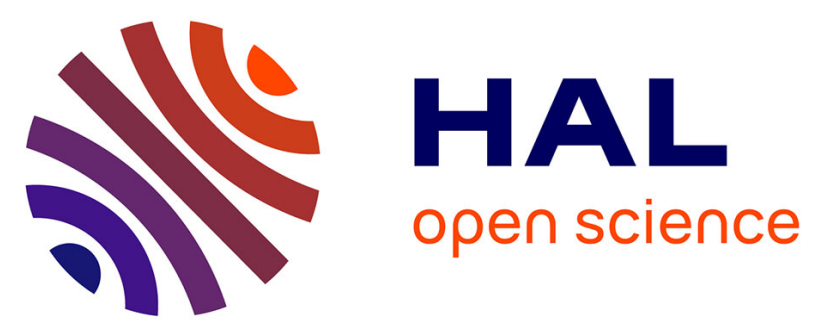

\title{
Kinetic theory of colloidal suspensions: morphology, rheology, and migration
}

Miroslav Grmela, Guillaume Maîtrejean, Francisco Chinesta, Amine Ammar

\section{To cite this version:}

Miroslav Grmela, Guillaume Maîtrejean, Francisco Chinesta, Amine Ammar. Kinetic theory of colloidal suspensions: morphology, rheology, and migration. Rheologica Acta, 2013, 52 (6), pp.557-577. 10.1007/s00397-013-0705-7 . hal-01207094

\section{HAL Id: hal-01207094 https://hal.science/hal-01207094}

Submitted on 30 Sep 2015

HAL is a multi-disciplinary open access archive for the deposit and dissemination of scientific research documents, whether they are published or not. The documents may come from teaching and research institutions in France or abroad, or from public or private research centers.
L'archive ouverte pluridisciplinaire HAL, est destinée au dépôt et à la diffusion de documents scientifiques de niveau recherche, publiés ou non, émanant des établissements d'enseignement et de recherche français ou étrangers, des laboratoires publics ou privés. 


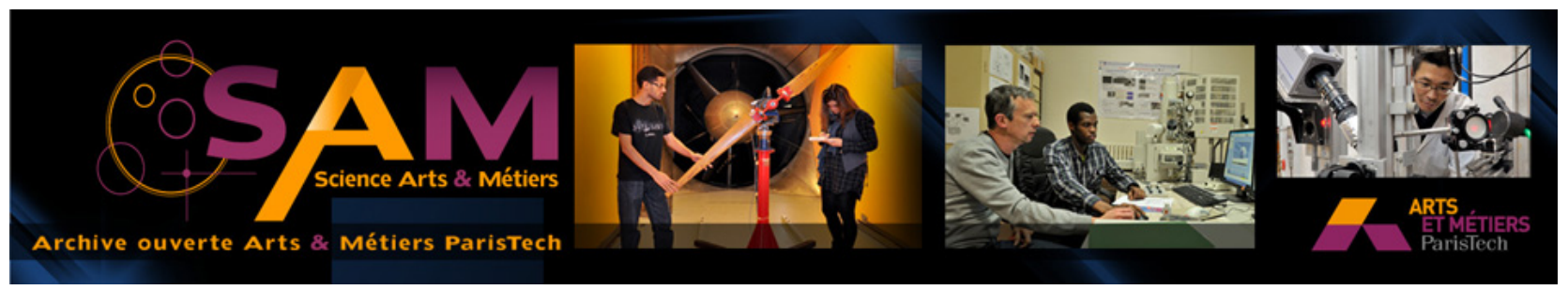

\section{Science Arts \& Métiers (SAM)}

is an open access repository that collects the work of Arts et Métiers ParisTech researchers and makes it freely available over the web where possible.

This is an author-deposited version published in: http://sam.ensam.eu

Handle ID: .http://hdl.handle.net/10985/10262

\section{To cite this version :}

Miroslav GRMELA, Guillaume MAîTREJEAN, Francisco CHINESTA, Amine AMMAR - Kinetic theory of colloidal suspensions: morphology, rheology, and migration - Rheologica Acta - Vol. 52, $n^{\circ} 6, p .557-577-2013$ 


\title{
Kinetic theory of colloidal suspensions: morphology, rheology, and migration
}

\author{
Miroslav Grmela • Guillaume Maîtrejean • \\ Francisco Chinesta $\cdot$ Amine Ammar
}

\begin{abstract}
Smoluchowski kinetic equation governing the time evolution of the pair correlation function of rigid spherical particles suspended in a Newtonian fluid is extended to include particle migration. The extended kinetic equation takes into account three types of forces acting on the suspended particles: a direct force generated by an interparticle potential, hydrodynamic force mediated by the host fluid, and the Faxén-type forces bringing about the across-the-streamline particle migration. For suspensions subjected to externally imposed flows, the kinetic equation is solved numerically by the proper generalized decomposition method. The imposed flow investigated in the numerical illustrations is the Poiseuille flow. Numerical solutions provide the morphology (the pair correlation function), the rheology (the stress tensor), and the particle migration.
\end{abstract}

\section{Grmela}

École Polytechnique de Montréal,

C.P. 6079 suc. Centre-ville, Montréal, H3C 3A7, Quebec, Canada e-mail: Miroslav.Grmela@polymtl.ca

\section{G. Maîtrejean ( $ه)$}

Mechanical and Materials Engineering Group, Engineering Department, European Organization for Nuclear Research (CERN), 1211 Geneva, Switzerland e-mail: Guillaume.Maitrejean@cern.ch

\section{F. Chinesta}

EADS Corporate Foundation International Chair,

Ecole Centrale de Nantes,

1 rue de la Noe, BP 92101, 44321 Nantes Cedex 3, France

e-mail: Francisco.Chinesta@ec-nantes.fr

\section{A. Ammar}

Arts et Métiers ParisTech,

2 Boulevard du Ronceray, BP 93525 ,

49035 Angers Cedex 01, France

e-mail: Amine.Ammar@ensam.eu
Keywords Suspension · Particle · Colloids · Modeling · Rheology

\section{Introduction}

Physical systems under consideration in this paper are semidilute isothermal suspensions of rigid spherical particles in Newtonian fluids. Externally imposed flows induce in such suspensions a fine-scale collective ordering and migration. The collective ordering manifests itself in the emergence of pair correlations and the migration in the emergence of spatial inhomogeneities in the distribution of suspended particles. Both the ordering and the migration as well as the resulting complex rheological behavior are investigated in this paper in the framework of kinetic theory. The same type of investigation, but without migration, has been reported in Zmievski et al. (2005) and Maîtrejean et al. (2012). In the rest of this section, we shall describe the strategy that we use in this paper to derive kinetic equations and recall the previously developed approaches to the investigation of the migration.

Multiscale theories of macroscopic systems

There are two distinct paths that can be followed in theoretical investigations of macroscopic systems. One, which we shall call bottom-up path, starts with a detailed microscopic description. The point of departure on the second path, which we shall call top-down path, is the requirement of agreement of solutions of the governing equations with results of certain basic experimental observations. Both paths are complementary and should be followed simultaneously. A combination of both paths then constitutes in fact a third path. 
In the case of the macroscopic systems investigated in this paper, the microscopic analysis involved in the bottomup path consists of a detailed microhydrodynamic analysis of the time evolution of all the suspended particles. Such analysis begins with identification of all the forces acting on the suspended particles and continues with writing down the corresponding Newton's equations and equations describing the time evolution of the fluid in which the particles are suspended. At this point, the path bifurcates into two branches. On the first one, called here direct simulation branch, the microscopic governing equations are solved with the assistance of computers. The final output is a set of trajectories of all suspended particles. An additional insight is then needed to reduce this complete microscopic information to the macroscopic information that can be compared with the information coming from macroscopic experimental observations. On the second branch, called here Liouville branch, the microscopic governing equations are first transformed into the Liouville equation that is then reduced to the Smoluchowski-type kinetic equation and to an expression for the extra stress tensor. The reduction processes on both branches consist of, in principle, a recognition of an overall pattern in the output of the complete microscopic analysis (that is the set of particle trajectories on the direct simulation branch and the set of solutions of the Liouville equation on the Liouville branch). Due to the lack of knowledge of the complete microscopic picture as well as due to the enormous difficulties involved in the process of pattern recognition, an additional insight and approximations are needed to make the micro-macro reductions. This is clearly a weak aspect of the bottom-up path. Its strong aspect is a very clear point of departure and a very clear microscopic meaning of the parameters entering the microscopic description.

On the top-down path, the governing equations are constructed as particular realizations of a mathematical structure guaranteeing that solutions to the equations possessing it have the required properties. By a particular realization, we mean that all the abstract elements of the structure (we call them modules) acquire a concrete meaning (as for example abstract elements of a group become matrices in group representations). Independently of what concretely is the mathematical structure (we shall discuss this question in the next paragraph), we see that the weak aspect of the top-down path is the phenomenological nature of the parameters entering the realizations and the strong aspect is the guaranteed intrinsic consistency and a very clear macroscopic meaning of the resulting governing equations. The bottom-up path and the top-down paths are thus indeed complementary.

Now, we turn to the mathematical structure involved in the top-down path. As we have already mentioned, we look for a mathematical structure that guarantees that solutions to equations possessing it agree with results of certain basic experimental observations. What are such observations? They are of two types: mechanical and thermodynamical. The former refers to observations of general consequences of Newton's law (as for example conservations of mass energy and momentum), and the latter refers to observations constituting the experimental basis of classical equilibrium thermodynamics (externally unforced macroscopic systems are seen to reach states, called thermodynamic equilibrium states, at which their behavior is seen to be well described by equilibrium thermodynamics). Two types of the mathematical structure have arisen in mesoscopic mechanics. First is the structure of local conservation laws (i.e., time derivative of a field equals divergence of a flux) originating in the Euler pioneering investigation (Euler 1755), and the second is the Hamiltonian structure originating in the Clebsch (1859) reformulation of the Euler equations into the Hamiltonian form. The former structure has been extended to include the thermodynamic observations in nonequilibrium thermodynamics (see, e.g., De Groot and Mazur 1984) and in Godunov (1961), Friedrichs and Lax (1971), Friedrichs (1978), and Godunov and Romenskii (2003) and the latter in Dzyaloshinskii and Volovick (1980), Grmela (1984a, b, 2010a, 2012a), Kaufman (1984), Morrison (1984), Beris and Edwards (1994), Grmela and Öttinger (1997), Öttinger and Grmela (1997), and Öttinger (2005). The disadvantage of the structure of local conservation laws is its limitation to settings that involve only partial differential equations, while its strong point is that the thermodynamic extension brings automatically a statement about mathematical regularity of the formulation (Godunov 1961; Friedrichs and Lax 1971; Friedrichs 1978; Godunov and Romenskii 2003). The strong point of the Hamiltonian structure is its applicability in a general setting (including, in particular, the setting of kinetic theory discussed in this paper) and its very clear thermodynamic interpretation (Legendre transformations, leading to the maximization of entropy, are made by the time evolution-see Grmela 2010a, 2012a). Among the recent applications of the structure, we mention dissipative quantum mechanics (Öttinger 2010), quantum fluids (Grmela 2010b), and chemical kinetics (Grmela 2012b; Klika and Grmela 2013).

In this paper, we derived the governing equations (see the Appendix) by using the top-down path based on the Hamiltonian structure. Every element of the structure (every modulus) is derived separately. Some of the modules, in particular the kinematics of state variables, are derived by following the bottom-up path. Some modules remain however to be specified only phenomenologically with only a partial support coming from microhydrodynamics. 


\section{Pair correlations and migration in suspensions}

In the "Governing equations" section, we present the governing equations and then investigate their solutions. The derivation of governing equations is presented in the Appendix. Here, we only briefly describe the essence of the physics involved in the derivation.

Experimental observations of suspensions of particles in simple non-Newtonian fluids show a complex rheological behavior and migration of particles from high to lower shear rates. If the particles are such that their shape and/or orientation can be influenced by imposed external flows, then this type of changes, characterized by changes in one-particle distribution function, plays a dominant role in determining the rheological responses. In addition, external forces can, at least in principle, induce a collective fine-structure ordering of the particles described by a pair correlation function and migration of particles creating inhomogeneity in their spatial distribution. An emergence of pair correlations and of their role in rheology of suspensions of fibers has been recently investigated in Grmela et al. (2012). If, on the other hand, the particles are spherical and rigid, it is only the flow-induced collective fine-structure ordering that can be responsible for the observed rheological complexity and the migration.

We shall first discuss the physics behind the pair correlations and then the physics behind the migration.

When following the bottom-up path, the microhydrodynamic origin of the flow-induced correlations among spherical particles is well understood (Batchelor and Green 1972; Batchelor 1977; Brady and Morris 1997) (for a recent review, see Nott et al. 2011). The hydrodynamic interactions (i.e., the interactions among suspended particles mediated by the host fluid), calculated by solving the corresponding Stokes problem, are responsible for the flow-induced correlations and consequently for the complex rheological behavior. In this paper, we arrive at flow-induced pair correlations of spherical particles by following the top-down approach. We show (in the Appendix) that the hydrodynamic interactions enter the top-down analysis as friction forces participating in driving the relative velocity of two particles to zero and the overall velocity to a uniform velocity. This type of derivation of the classical Smoluchowski equation governing the time evolution of the pair correlation function is the first original contribution of this paper.

The physics behind the microhydrodynamic origins of the migration is much less known (for a recent review, see Nott et al. 2011). An interesting new insight into this subject has appeared in Lhuillier (2009) (see also Nott et al. 2011 where this new insight is put into the context of previous investigations). Lhuillier has suggested that the Faxén forces are among the forces that drive the migration. Faxén forces arise in microhydrodynamics in attempts to carry the standard analysis of the Stokes problem to situations involving large inhomogeneities in the shear rate. These forces are proportional to the second derivatives of the flow velocity with respect to the position coordinate. Lhuillier has included Faxén forces in a continuum mechanics formulation in which the suspension is seen as a two-phase fluid. In this paper, we include the Faxén forces in a kinetic theory formulation developed by using the top-down approach. We show that:

1. In the context of this approach to kinetic theory, the Faxén forces enter as friction forces, participating in bringing to zero the difference between the translational velocity of the center of mass of two particles and the overall velocity of the suspension and in bringing the overall velocity to a velocity with a uniform gradient.

2. The Faxén forces indeed cause particle migration observed in experimental observations.

The latter result appears in numerical solutions (see the "Results" section) of the Smoluchowski equation modified by the presence of Faxén forces. We make no contribution in this paper to the way the Faxén forces arise in microhydrodynamics and to their connection (inside the microhydrodynamic analysis) with particle migration.

\section{Numerical method}

Having the kinetic equation, we have to solve it in order to be able to obtain results that can be compared with the results of experimental observations and results arising in other theoretical approaches. Since the distribution function (the function that we look for as a solution of the kinetic equation) is a function of seven independent variables in $3 \mathrm{D}$ (five in the case of 2D), the task of solving the kinetic equation appears to be prohibitively difficult with standard numerical methods. The recently developed proper generalized decomposition (PGD) method (Ammar et al. 2006, 2007, 2009; Chinesta et al. 2010, 2011) makes the task feasible as it has already been demonstrated in Maîtrejean et al. (2012). The method can be seen as a numerical version of the method of separation of variables.

\section{Governing equations}

In this section, we set up the kinetic theory of moderately concentrated suspensions of hard spherical particles. The new physics that is put into the governing equations is the physics behind the Faxén forces causing migration of suspended particles. Without the Faxén forces, the kinetic 
equation arising in this paper is exactly the same as the classical Smoluchowski equation investigated in Zmievski et al. (2005) and Maîtrejean et al. (2012). In order to see clearly how the new physics expressed in Faxén forces modifies the kinetic equation, we present its derivation in the Appendix.

Kinetic equation

The Smoluchowski-type kinetic equation, expressing mathematically in this paper the physics of suspensions of rigid spherical particles, is presented as follows:

$$
\begin{aligned}
\frac{\partial g}{\partial t}= & -\frac{\partial}{\partial r_{j}}\left(g \Phi_{u_{j}}\right) \\
& +\frac{\partial}{\partial r_{j}}\left[g\left(\kappa_{i j}^{(\mathrm{I})} \mathcal{Y}_{j}^{(\mathrm{I})}+\kappa_{i j}^{(\mathrm{II})} \mathcal{Y}_{j}^{(\mathrm{II})}+\kappa_{i j}^{(\mathrm{III})} \mathcal{Y}_{j}^{(\mathrm{III})}\right)\right] \\
& -\frac{\partial}{\partial R_{j}}\left(g R_{k} \frac{\partial \Phi_{u_{j}}}{\partial r_{k}}\right)+\frac{\partial}{\partial R_{j}}\left(g \lambda_{j k} R_{l} D_{k l}\right) \\
& +\frac{\partial}{\partial r_{i}}\left(g \kappa_{i j} \frac{\partial \Phi_{g}}{\partial r_{j}}\right)+\frac{\partial}{\partial R_{i}}\left(g \Lambda_{i j} \frac{\partial \Phi_{g}}{\partial R_{j}}\right) .
\end{aligned}
$$

$g(\boldsymbol{r}, \boldsymbol{R}, t)$ denotes a pair correlation function, $\boldsymbol{r} \in \mathbb{R}^{3}$ stands for the position vector, $R \in \mathbb{R}^{3}$ is the vector connecting two suspended particles, and $t$ is the time. The pair correlation function is normalized so that

$$
\int d \boldsymbol{R} g(\boldsymbol{r}, \boldsymbol{R}, t)=n_{\mathrm{p}}(\boldsymbol{r}, t)
$$

where $n_{\mathrm{p}}(\boldsymbol{r}, t)$ is the number density of suspended particles. The other symbols appearing in Eq. 1 have the following meaning: $D_{i j}=\frac{1}{2}\left(\frac{\partial \Phi_{u_{i}}}{\partial r_{j}}+\frac{\partial \Phi_{u_{j}}}{\partial r_{i}}\right), \mathcal{Y}_{i}^{(\mathrm{I})}=R^{2} \mathcal{D}_{j j i}, \mathcal{Y}_{i}^{(\mathrm{II})}=$ $R_{j} R_{k} \mathcal{D}_{i j k}$, and $\mathcal{Y}_{i}^{\text {(III) }}=R_{i} R_{k} \mathcal{D}_{j j k}$, where the symbol $\mathcal{D}_{i j k}$ stands for $\mathcal{D}_{i j k}=\frac{1}{3}\left(\frac{\partial D_{j k}}{\partial r_{i}}+\frac{\partial D_{i k}}{\partial r_{j}}+\frac{\partial D_{i j}}{\partial r_{k}}\right) ; \Phi$ is a free energy specified below; and $\lambda, \boldsymbol{\Lambda}, \boldsymbol{\kappa}, \boldsymbol{\kappa}^{(\mathrm{I})}, \boldsymbol{\kappa}^{(\mathrm{II})}$, and $\boldsymbol{\kappa}^{(\mathrm{III})}$ are material parameters that are also specified below. We use hereafter the following shorthand notations: $\Phi_{u_{i}}=\frac{\partial \Phi}{\partial u_{i}(\boldsymbol{r})}$ and $\Phi_{g}=\frac{\partial \Phi}{\partial g(\boldsymbol{r}, \boldsymbol{R})}$. For the sake of the simplicity of the notation, we use the symbol $\partial$ for the usual partial derivatives and also for the functional derivatives. We also use in this paper the Einstein summation convention.

The first term on the right-hand side of Eq. 1 expresses passive advection in the $\boldsymbol{r}$-space, the second term expresses the advection also in the $\boldsymbol{r}$-space due to Faxén forces, and the third and the fourth terms represent advection in the $\boldsymbol{R}$ space due to hydrodynamic interaction. Finally, the fifth and the sixth terms represent dissipation, with the fifth term representing the standard Fickian diffusion in the $\boldsymbol{r}$-space and the sixth term representing the diffusion in the $\boldsymbol{R}$-space.

We note that if all the parameters $\boldsymbol{\kappa}$ are equal to zero (i.e., if the Faxén forces disappear), then the kinetic equation (1) becomes the classical Smoluchowski kinetic equation discussed in Zmievski et al. (2005) and Maîtrejean et al. (2012).

\section{Stress tensor}

In order to be able to address the rheological behavior of the suspensions, we have to know how the extra stress tensor (observed in rheological measurements) is expressed in terms of the morphology characterized by the pair correlation function. The formula

$$
\begin{aligned}
\sigma_{j k}=\int d \boldsymbol{R}[- & g R_{k} \frac{\partial \Phi_{g}}{\partial R_{j}}+g \lambda_{m k} R_{j} \frac{\partial \Phi_{g}}{\partial R_{m}} \\
& -\frac{2}{3} \kappa^{(\mathrm{I})} R^{2} \frac{\partial}{\partial r_{k}}\left(g \frac{\partial \Phi_{g}}{\partial r_{j}}\right) \\
& -\frac{1}{3} \kappa^{(\mathrm{II})} R_{j} R_{k} \frac{\partial}{\partial r_{m}}\left(g \frac{\partial \Phi_{g}}{\partial r_{m}}\right) \\
& -\frac{1}{3} \kappa^{(\mathrm{II})} R_{k} R_{m} \frac{\partial}{\partial r_{m}}\left(g \frac{\partial \Phi_{g}}{\partial r_{j}}\right) \\
& -\frac{1}{3} \kappa^{(\mathrm{II})} R_{j} R_{m} \frac{\partial}{\partial r_{m}}\left(g \frac{\partial \Phi_{g}}{\partial r_{k}}\right) \\
& -\frac{1}{3} \kappa^{(\mathrm{III})} R_{k} R_{m} \frac{\partial}{\partial r_{j}}\left(g \frac{\partial \Phi_{g}}{\partial r_{m}}\right) \\
& \left.-\frac{1}{3} \kappa^{(\mathrm{IIII})} R_{j} R_{m} \frac{\partial}{\partial r_{k}}\left(g \frac{\partial \Phi_{g}}{\partial r_{m}}\right)\right]
\end{aligned}
$$

that we use for this purpose in this paper is derived in the Appendix. By $\boldsymbol{\sigma}$, we denote the extra stress tensor.

We observe again that without the terms proportional to the parameters $\kappa$, the formula (3) is the same as the one used in Maîtrejean et al. (2012).

Free energy

The free energy $\Phi$ is chosen in this paper to be exactly the same as in Zmievski et al. (2005) and Maîtrejean et al. (2012):

$$
\begin{aligned}
\Phi= & \int d \boldsymbol{r} \frac{\boldsymbol{u}^{2}}{2 \rho}+\int d \boldsymbol{r} \int d \boldsymbol{R} g(\boldsymbol{r}, \boldsymbol{R})(U(\boldsymbol{R}) \\
& \left.-k_{\mathrm{B}} T \ln g(\boldsymbol{r}, \boldsymbol{R})\right) \\
U= & U_{\mathrm{HC}}+U_{\mathrm{nl}} \\
U_{\mathrm{HC}}(R)= & 0 \text { for } R \geq 2 a \text { and } \infty \text { for } R<2 a \\
U_{\mathrm{nl}}(R)= & \frac{\epsilon}{R^{12}} \text { for } R \geq 2 a .
\end{aligned}
$$

$R=|\boldsymbol{R}|, a$ is the radius of the suspended particles, $\epsilon$ is a small positive constant, $k_{\mathrm{B}}$ is the Boltzmann constant, and $T$ is the temperature (assumed to be constant). The potential $U_{\mathrm{HC}}$ is the hard-core potential and the potential $U_{\mathrm{nl}}$ is added 
in order to make the singularity at $R=2 a$ manageable in numerical calculations. The first term on the right-hand side of the first equation in Eq. 4 represents the kinetic energy of the overall motion and the third term represents the entropy.

We note that the pair correlation function $g_{\text {eq }}$ corresponding to the equilibrium state (i.e., the state for which the free energy $\left.\Phi_{g}=0\right)$ is $g_{\text {eq }}=K e^{\frac{U_{\mathrm{nl}}}{k_{\mathrm{B}} T}}$, where $K$ is the normalization constant (see more in Zmievski et al. 2005).

\section{Material parameters}

Microhydrodynamic investigations of the hydrodynamic interaction between two spheres suspended in a host fluid have led Batchelor and Green (1972) to

$$
\begin{aligned}
& \Lambda_{i j}=\frac{k_{\mathrm{B}} T}{3 \pi \eta_{0} a}\left[\sigma_{1}(R / a) \frac{R_{i} R_{j}}{R^{2}}+\sigma_{2}(R / a)\left(\delta_{i j}-\frac{R_{i} R_{j}}{R^{2}}\right)\right] \\
& \lambda_{i j}=\mu_{1}(R / a) \frac{R_{i} R_{j}}{R^{2}}+\mu_{2}(R / a)\left(\delta_{i j}-\frac{R_{i} R_{j}}{R^{2}}\right)
\end{aligned}
$$

where $\eta_{0}$ is the viscosity coefficient of the host fluid and the coefficients $\sigma_{1}, \sigma_{2}, \mu_{1}$, and $\mu_{2}$ are given approximatively (see Zmievski et al. 2005) by

$\sigma_{1} \approx 1-\frac{3}{2} \frac{a}{R}+\frac{a^{3}}{R^{3}}-\frac{15}{4} \frac{a^{4}}{R^{4}}+109.0 \frac{a^{6}}{R^{6}}-236.0 \frac{1}{R^{7}}$,

$\sigma_{2} \approx 1-\frac{3}{4} \frac{a}{R}-\frac{1}{2} \frac{a^{3}}{R^{3}}-10.336 \frac{a^{6}}{R^{6}}$

and

$\mu_{1} \approx 5 \frac{a^{3}}{R^{3}}-8 \frac{a^{5}}{R^{5}}+25 \frac{a^{6}}{R^{6}}-423.712 \frac{a^{7}}{R^{7}}+907.424 \frac{a^{8}}{R^{8}}$,

$\mu_{2} \approx \frac{16}{3} \frac{a^{5}}{R^{5}}+15.317 \frac{a^{6}}{R^{6}}$.

We used these expressions from Zmievski et al. (2005) and Maîtrejean et al. (2012).

Phenomenological considerations reported in Lhuillier (2009) have led us to supplement the thermodynamic forces $\mathcal{X}$ bringing about the hydrodynamic interactions by the Faxén-type forces $\mathcal{Y}$ which bring about the across-thestreamline migration of the suspended particles (see the Appendix). For the sake of simplicity, we shall consider the matrices $\boldsymbol{\kappa}$ to be just scalars, i.e.,

$$
\kappa_{i j}^{(\mathrm{I})}=\kappa^{(\mathrm{I})} \delta_{i j} ; \kappa_{i j}^{(\mathrm{II})}=\kappa^{(\mathrm{II})} \delta_{i j} ; \kappa_{i j}^{(\mathrm{III})}=\kappa^{(\mathrm{III})} \delta_{i j} .
$$

Dimensionless form of the governing equations

The transformation of the governing equations to their dimensionless forms is chosen to be the same as in Zmievski et al. (2005) and Maîtrejean et al. (2012):

$$
\begin{aligned}
& t \rightarrow t / \tau_{\mathrm{S}} ; \boldsymbol{r} \rightarrow \boldsymbol{r} / a ; \boldsymbol{R} \rightarrow \boldsymbol{R} / a ; U_{\mathrm{nl}} \rightarrow \frac{U_{\mathrm{nl}}}{k_{\mathrm{B}} T} \\
& \boldsymbol{u} \rightarrow \boldsymbol{u} / u_{0} ; \boldsymbol{\sigma} \rightarrow \frac{\boldsymbol{\sigma}}{\eta_{0} \dot{\gamma}} ; \boldsymbol{\Lambda} \rightarrow \frac{3 \pi \eta_{0} a}{k_{\mathrm{B}} T} \boldsymbol{\Lambda}
\end{aligned}
$$

where $\eta_{0}$ is the viscosity coefficient of the host fluid, $\tau_{\mathrm{S}}=$ $\frac{6 \pi \eta_{0} a^{3}}{k_{\mathrm{B}} T}$ is the Smoluchowski characteristic relaxation time, and $u_{0}=\frac{\rho a}{\tau_{\text {hyd }}}$, where $\tau_{\text {hyd }}=(\dot{\gamma})^{-1}$ is the hydrodynamic characteristic time. Moreover, we introduce two dimensionless numbers: the Reynolds number mathrm Re $=\frac{u_{0} a}{\eta_{0}}$ and the Péclet number Pe $=\frac{\tau_{\mathrm{S}}}{\tau_{\text {hyd }}}$. By $\triangle g$, we denote $g-g_{\text {eq }}$, $\boldsymbol{n}=\frac{\boldsymbol{R}}{R}$, and $\int_{S_{2}} d \boldsymbol{n}$ denotes integration over the unit sphere.

The dimensionless kinetic equation (1) takes the following form:

$$
\begin{aligned}
\frac{\partial g}{\partial t}= & -\mathrm{Pe} \frac{\partial\left(g u_{j}\right)}{\partial r_{j}}+\operatorname{Pe} \frac{\partial}{\partial r_{j}} \\
& {\left[g\left(\kappa^{(\mathrm{I})} \mathcal{Y}_{j}^{(\mathrm{I})}+\kappa^{(\mathrm{II})} \mathcal{Y}_{j}^{(\mathrm{II})}+\kappa^{(\mathrm{III})} \mathcal{Y}_{j}^{(\mathrm{III})}\right)\right] } \\
& -\mathrm{Pe} \frac{\partial}{\partial R_{j}}\left(R_{k} \frac{\partial u_{j}}{\partial r_{k}} \Delta g\right)+\operatorname{Pe} \frac{\partial}{\partial R_{j}}\left(g \lambda_{j k} \mathcal{X}_{k}\right) \\
& +\frac{\partial}{\partial r_{i}}\left(\kappa \frac{\partial g}{\partial r_{i}}\right)+\frac{\partial}{\partial R_{j}}\left(g_{\text {eq }} \Lambda_{j k} \frac{\partial}{\partial R_{k}}\left(\frac{g}{g_{\text {eq }}}\right)\right),
\end{aligned}
$$

and the dimensionless expression for $\sigma$ (see Eq. 3) reads

$$
\begin{aligned}
\sigma_{j k}= & -\left(1+\frac{2}{5} \phi\right) D_{j k}-\frac{27}{8 \pi} \frac{\phi^{2}}{\mathrm{Pe}} \\
& \left\{2 \int_{S_{2}} d \boldsymbol{n}\left(n_{j} n_{k}-\frac{1}{3} \delta_{j k}\right)\left(1-\mu_{1}(2)\right) \triangle g(2 \boldsymbol{n})\right. \\
& -\int d \boldsymbol{R}\left[3\left(\mu_{1}-\mu_{2}\right)+R \frac{\partial \mu_{1}}{\partial \boldsymbol{R}}+\left(1-\mu_{1}\right) R \frac{\partial U_{\mathrm{nl}}}{\partial \boldsymbol{R}}\right] \\
& \times\left(n_{j} n_{k}-\frac{1}{3} \delta_{j k}\right) \triangle g(\boldsymbol{R}) \\
& +\int d \boldsymbol{R}\left[-\frac{2}{3} \kappa^{(\mathrm{I})} R^{2} \frac{\partial}{\partial r_{k}}\left(\frac{\partial g}{\partial r_{j}}\right)-\frac{1}{3} \kappa^{(\mathrm{III})} R_{j} R_{k} \frac{\partial}{\partial r_{m}}\left(\frac{\partial g}{\partial r_{m}}\right)\right. \\
& -\frac{1}{3} \kappa^{(\mathrm{II})} R_{m} R_{k} \frac{\partial}{\partial r_{m}}\left(\frac{\partial g}{\partial r_{j}}\right)-\frac{1}{3} \kappa^{(\mathrm{II})} R_{j} R_{m} \frac{\partial}{\partial r_{m}}\left(\frac{\partial g}{\partial r_{k}}\right) \\
& \left.\left.\frac{1}{3} \kappa^{(\mathrm{III})} R_{m} R_{k} \frac{\partial}{\partial r_{j}}\left(\frac{\partial g}{\partial r_{m}}\right)-\frac{1}{3} \kappa^{(\mathrm{III})} R_{j} R_{m} \frac{\partial}{\partial r_{k}}\left(\frac{\partial g}{\partial r_{m}}\right)\right]\right\} .
\end{aligned}
$$

Both Eqs. 10 and 11 reduce to the corresponding equations appearing in Maîtrejean et al. (2012) if the parameters $\kappa$ are equal to zero. 
Boundary conditions and the weak form of the kinetic equation

In the $\boldsymbol{R}$-space, we choose the same boundary conditions as in Zmievski et al. (2005) and Maîtrejean et al. (2012) (i.e., $g \rightarrow 1$ as $R \rightarrow \infty$, and on the boundary $R=2 a$, we require that the flow of the particles, in the direction perpendicular to it, vanishes). In the $\boldsymbol{r}$-space, we assume that $g=1$ on its boundary. With these boundary conditions, the weak form of the kinetic equation (the form that is then discretized by using the standard finite element procedure and subjected further to the separation procedure used in the PGD method-for details, see Maîtrejean et al. 2012) reads

$$
\begin{aligned}
\int_{\omega \times \Omega} d \boldsymbol{r} d \boldsymbol{R}\left(g^{*}\right. & \frac{\partial g}{\partial t}+g^{*} \boldsymbol{v} \frac{\partial g}{\partial \boldsymbol{r}}+g^{*} E_{0} g-g^{*} \boldsymbol{E}_{1} \frac{\partial g}{\partial \boldsymbol{r}} \\
& +g^{*} \boldsymbol{E}_{2} \frac{\partial g}{\partial \boldsymbol{R}}+\frac{\partial g^{*}}{\partial \boldsymbol{r}} \boldsymbol{E}_{3} \frac{\partial g}{\partial \boldsymbol{r}} \\
& \left.+\frac{\partial g^{*}}{\partial \boldsymbol{R}} \boldsymbol{E}_{4} \frac{\partial g}{\partial \boldsymbol{R}}+\frac{\partial g^{*}}{\partial \boldsymbol{R}} \boldsymbol{E}_{5} g\right) \\
& -\int_{\omega \times \Gamma} d \boldsymbol{r} d \boldsymbol{R} g^{*}\left(\boldsymbol{n} \cdot \boldsymbol{E}_{2}\right) g \\
& =\int_{\omega \times \Omega} d \boldsymbol{r} d \boldsymbol{R} g^{*} F
\end{aligned}
$$

where $\boldsymbol{n}=\boldsymbol{R} / R, \boldsymbol{r} \in \omega, \boldsymbol{R} \in \Omega, \int_{\Gamma} d \boldsymbol{R}$ denotes integration over the boundary $\Gamma$ of $\Omega$, and

$$
\begin{aligned}
E_{0}(\boldsymbol{r}, \boldsymbol{R})= & \operatorname{Pe}\left(\frac{\partial v_{i}}{\partial r_{i}}+\frac{\partial R_{k}}{\partial R_{j}} \frac{\partial v_{j}}{\partial r_{k}}\right) \\
& -\operatorname{Pe}\left(\frac{\partial \lambda_{j l}}{\partial R_{j}} \mathcal{X}_{k}+\lambda_{j k} \frac{\partial \mathcal{X}_{k}}{\partial R_{j}}\right) \\
\boldsymbol{E}_{1}(\boldsymbol{r}, \boldsymbol{R})= & \kappa^{(\mathrm{I})} \mathcal{Y}^{(\mathrm{I})}+\kappa^{(\mathrm{II})} \mathcal{Y}^{(\mathrm{II})}+\kappa^{(\mathrm{III})} \mathcal{Y}^{(\mathrm{III})} \\
\boldsymbol{E}_{3}(\boldsymbol{R})= & \kappa \boldsymbol{\delta} \\
\boldsymbol{E}_{4}(\boldsymbol{R})= & \boldsymbol{\Lambda} \\
\boldsymbol{E}_{5}(\boldsymbol{R})= & \boldsymbol{\Lambda} g_{\mathrm{eq}} \frac{\partial}{\partial \boldsymbol{R}}\left(\frac{1}{g_{\mathrm{eq}}}\right) \\
F(\boldsymbol{r}, \boldsymbol{R})= & \operatorname{Pe}\left(\frac{\partial R_{k}}{\partial R_{j}} \frac{\partial u_{j}}{\partial r_{k}} g_{\mathrm{eq}}+R_{k} \frac{\partial}{\partial R_{j}}\left(\frac{\partial u_{j}}{\partial r_{k}}\right) g_{\mathrm{eq}}\right. \\
& \left.\quad+R_{k} \frac{\partial u_{j}}{\partial r_{k}} \frac{\partial g_{\mathrm{eq}}}{\partial R_{j}}\right) .
\end{aligned}
$$

This formulation directly extends (by including the terms proportional to the parameters $\boldsymbol{\kappa}$ ) the formulation appearing in Eqs. 23-28 in Maîtrejean et al. (2012).

In the calculations leading to Eq. 12, we have neglected the derivatives of $\mathcal{Y}^{(\mathrm{I})}, \mathcal{Y}^{(\mathrm{II})}$, and $\mathcal{Y}^{\text {(III) }}$ with respect to $\boldsymbol{r}$ (involving third derivatives of $\boldsymbol{u}(\boldsymbol{r})$ with respect to $\boldsymbol{r}$ ).

\section{Results}

As the next step, we have to solve the kinetic equation (10) in order to be able to see the morphology, the rheology, and the migration that it implies. This is a rather difficult task. In fact, due to the high dimensionality (seven in 3D and five in 2D) of the variables on which we search the function $g(\boldsymbol{r}, \boldsymbol{R}, t)$ as a solution of the kinetic equation, the task is practically unfeasible if approached with traditional numerical methods. The dimensionality can be reduced by passing to moments (in particular, in the $\boldsymbol{R}$-variable). This strategy has been used for the Smoluchowski kinetic equation in Zmievski et al. (2005). The disadvantage of this approach is that the dimensionality becomes reduced only if a finite number of moments remains in the formulation. But for this to be the case, one needs a closure (i.e., one needs to know how to express all the higher moments in terms of the finite number moments chosen to be explicitly followed). An additional new physical insight is needed to specify closures. The physics expressed in the original kinetic equation becomes therefore, at least in general, different from the physics that is expressed in its finite-moment reformulation. Consequently, we prefer to avoid the moment method.

It has been demonstrated in Maîtrejean et al. (2012) that with the PGD method, which was recently developed by Ammar et al. $(2006,2007,2009)$ and Chinesta et al. (2010, 2011), the problem of solving numerically the Smoluchowski kinetic equation becomes feasible without restoring to moments and closures. The new feature of the kinetic equation appearing in this paper, namely the presence of additional terms involving higher order derivatives with respect to the $\boldsymbol{r}$-coordinate, makes the numerical calculations longer but the PGD method remains perfectly applicable. For details on how the PGD method is applied to the Smoluchowski-type kinetic equations, we refer to Maîtrejean et al. (2012).

Poiseuille flow

Migration across the streamlines is not observed in simple shear flows with a constant shear rate. This indeed agrees with predictions of Eq. 1 since the Faxén forces $\mathcal{Y}$ all vanish for the flows with a constant velocity gradient. In order to illustrate the migration and the morphology and rheology associated with it, we turn therefore to another very familiar flow, namely the Poiseuille flow. In two dimensions, the case to which we restrict ourselves in this paper, the imposed velocity field, is given by

$\boldsymbol{v}=\left(\begin{array}{c}1-\frac{y^{2}}{h^{2}} \\ 0\end{array}\right)$

where $\boldsymbol{r}=(x, y)^{T}$. 
Fig. 1 The domain $\omega$ and the Poiseuille velocity field (represented by the arrows)

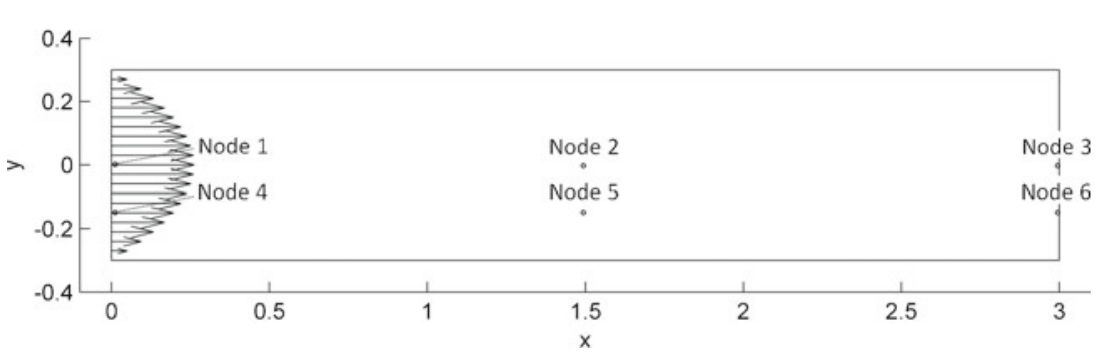

Following the PGD method, we look for a solution of the modified Smoluchowski equation (10) with the velocity gradient (14) in the form

$$
g(\boldsymbol{r}, \boldsymbol{R})=\sum_{i=1}^{n_{g}} \mathcal{F}^{i}(x) \otimes \mathcal{F}^{i}(y) \otimes \mathcal{F}^{i}(\boldsymbol{R}) .
$$

The $x, y$, and $\boldsymbol{R}$-spaces are discretized with 41,201 , and 1,680 nodes. The points $\boldsymbol{r}_{i}, i=1, \ldots, 6$, referred hereafter as nodes 1 to 6 are depicted in Fig. 1. At these nodes, we shall be presenting below the $\boldsymbol{R}$ dependence of the correlation function $g$.

The boundary conditions are the following: $g=1$ for $R>5$ for all $r$ and $g=1$ at the entrance and on the top wall (see Fig. 1).

Below, we shall present solutions of Eq. 10 for $\epsilon=0$ appearing in Eq. 4, for the Péclet number $\mathrm{Pe}=1$, and for a selection of the parameters $\kappa, \kappa^{(\mathrm{I})}, \kappa^{(\mathrm{II})}$, and $\kappa^{(\mathrm{III})}$.

\section{No Faxén forces}

We begin with the case when all the parameters $\kappa$ are equal to zero. In this case, the modified Smoluchowski Eq. 10 becomes the classical Smoluchowski equation. In Fig. 2, we present $n_{\mathrm{p}}(\boldsymbol{r})=\int d \boldsymbol{R} g(\boldsymbol{r}, \boldsymbol{R})$, where $g(\boldsymbol{r}, \boldsymbol{R})$ is the steady-state solution of the classical Smoluchowski equation.

We note that the distance for $n_{\mathrm{p}}(\boldsymbol{r})$ to reach its maximum depends on the velocity gradient: near the top wall, where the velocity gradient is high, we observe a short distance between the flow inlet and the maximum. On the other hand, at the center (i.e., the bottom line), the velocity gradient vanishes and the pair correlation remains uncorrelated.

\section{Diffusion in the $\boldsymbol{r}$-space $(\kappa \neq 0)$}

The next case that we investigate corresponds to $\kappa \neq 0$ (i.e., the diffusion in the $r$-space is taken into account) but $\kappa^{(\mathrm{I})}=$
$\kappa^{(\mathrm{II})}=\kappa^{(\mathrm{III})}=0$ (i.e., the Faxén forces are absent). We choose $\kappa=\frac{\alpha}{R^{2}}$.

Figure 3 depicts steady-state solutions of Eq. 10. We no longer observe an uncorrelated zone at the center. This means that the migration of the particles begins to occur. We also observe a competition between the shear and the diffusion forces. In the $\boldsymbol{R}$-space, i.e., in the microscale ordering, the influence of the diffusion is seen in the amplitude of the pair correlation function. The orientation of correlations remains the same for all values of $\alpha$, only the distance between the particles varies (Fig. 4).

Influence of $\kappa^{(\mathrm{I})}, \kappa^{(\mathrm{II})}$, and $\kappa^{(\mathrm{III})}$

Now we proceed to investigate the influence of the parameters $\kappa^{(\mathrm{I})}, \kappa^{(\mathrm{II})}$, and $\kappa^{(\mathrm{III})}$. In this investigation, we have to still keep the diffusion in the $r$-space (i.e., $\kappa \neq 0$ and thus $\alpha \neq 0$ ) in order to preserve the numerical stability (note that the terms proportional to $\kappa^{(\mathrm{I})}, \kappa^{(\mathrm{II})}$, and $\kappa^{(\mathrm{III})}$ are convective terms) (Figs. 5, 6, 7, 8, 9, and 10).

We shall comment about these results below in the context of the comparison with results of experimental observations.

\section{Comparison with experimental results}

The modified Smoluchowski (Eq. 1) takes into account the migration but remains still limited to rather dilute suspensions. It has been shown in Maîtrejean et al. (2012) that solutions to the original Smoluchowski equation are in good agreement with the results of numerical simulations based on the microhydrodynamic formulation reported in Brady and Morris (1997) in simple flows. However, despite a good agreement in the orientation of the pair correlation function in the $\boldsymbol{R}$-space, the results differ in magnitude. This difference has been attributed in Maîtrejean et al. (2012) to the fact that the suspension investigated in Brady and Morris (1997) is dense $(\phi=0.3)$.

Fig. 2 Steady state of $n_{\mathrm{p}}(\boldsymbol{r})$

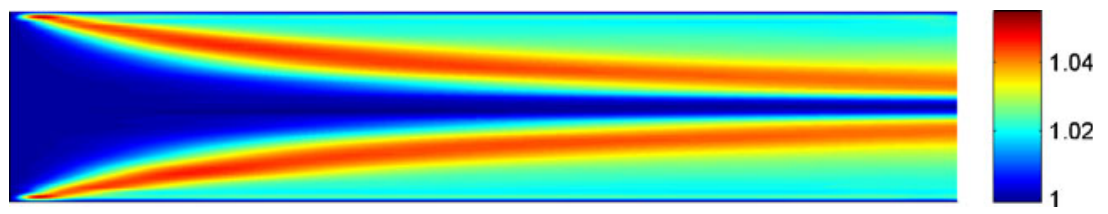


Fig. 3 Steady state of $n_{\mathrm{p}}(\boldsymbol{r})$ and $g$ at nodes 1 to 6 with $\alpha=0.01$

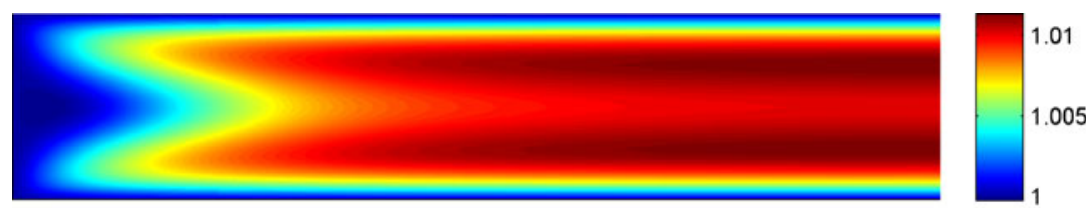

(a) $n_{p}(r)$

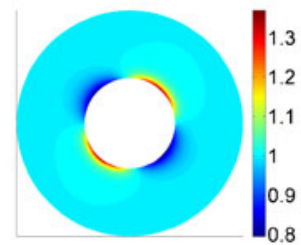

(b) $g(r, R)$ at node 1

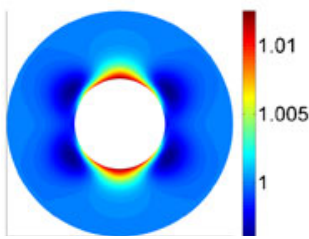

(e) $g(r, R)$ at node 4

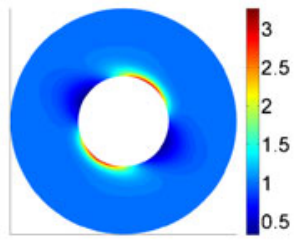

(C) $g(r, R)$ at node 2

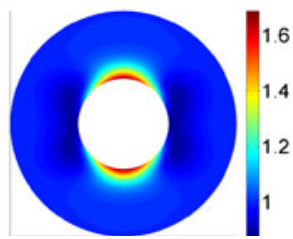

(f) $g(r, R)$ at node 5

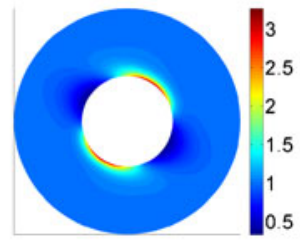

(d) $g(\boldsymbol{r}, \boldsymbol{R})$ at node 3

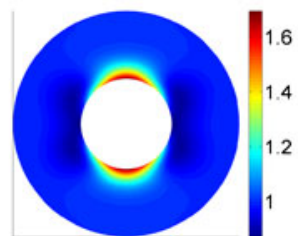

(g) $g(r, R)$ at node 6
Also, in the case of particle migration, most of the experimental work and the microhydrodynamic simulations have been done for dense suspensions. As noted in Frank et al. (2003), the effect of the particle migration in colloidal suspensions scales as $\phi^{2}$. Consequently, for dilute suspensions, the migration is weak and its is difficult to observe it experimentally. Nevertheless, the local maxima of the particle density appear to be clearly reproducible.

With this in mind, we compare the results implied by the modified Smoluchowski model (1) to the experimental results reported in Frank et al. (2003). The experimental
Fig. 4 Steady state of $n_{\mathrm{p}}(\boldsymbol{r})$ and $g$ at nodes 1 to 6 with $\alpha=0.1$
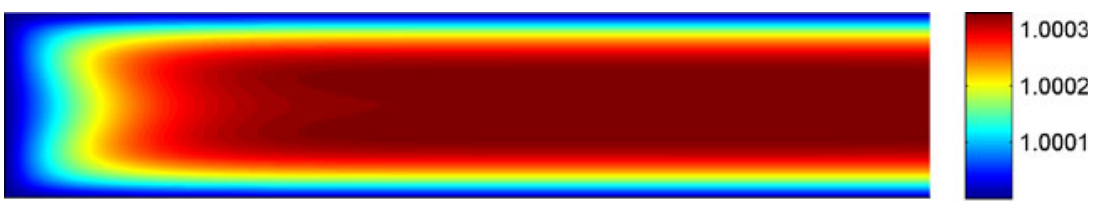

(a) $n_{p}(r)$

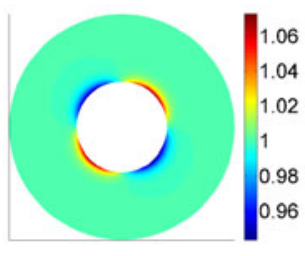

(b) $g(r, R)$ at node 1

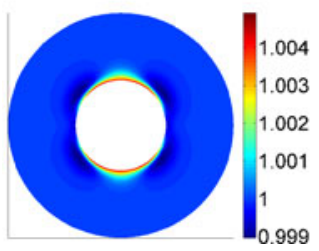

(e) $g(r, R)$ at node 4

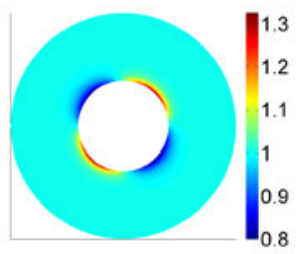

(C) $g(r, R)$ at node 2

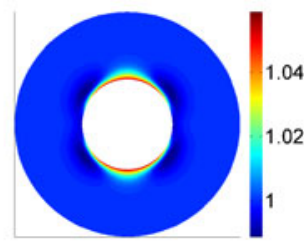

(f) $g(r, R)$ at node 5

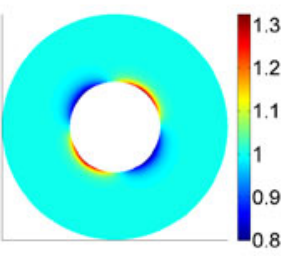

(d) $g(\boldsymbol{r}, \boldsymbol{R})$ at node 3

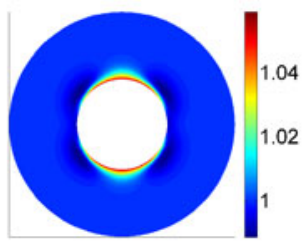

(g) $g(\boldsymbol{r}, \boldsymbol{R})$ at node 6 
Fig. 5 Steady state of $n_{\mathrm{p}}(\boldsymbol{r})$ and $g$ at nodes 1 to 6 with $\kappa^{(\mathrm{I})}=\frac{0.5}{\boldsymbol{R}^{2}}$

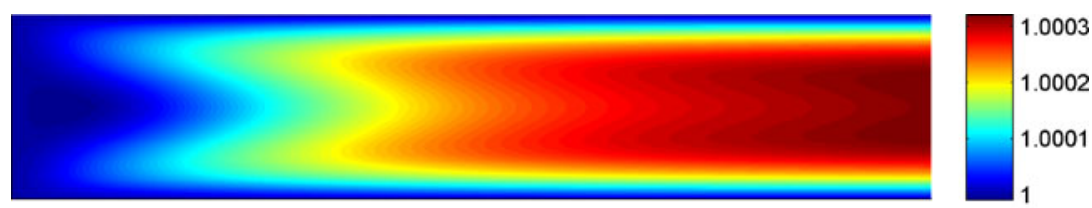

(a) $\mathrm{n}_{\mathrm{p}}(\boldsymbol{r})$

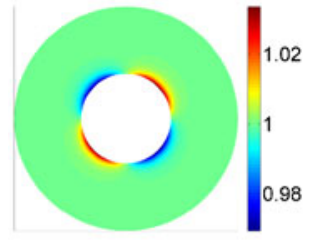

(b) $g(r, R)$ at node 1

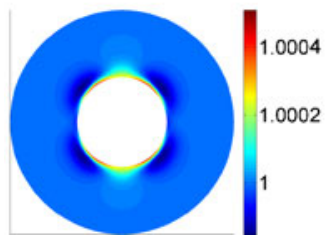

(e) $g(r, R)$ at node 4

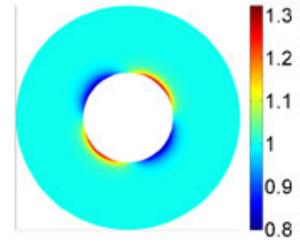

(C) $g(r, R)$ at node 2

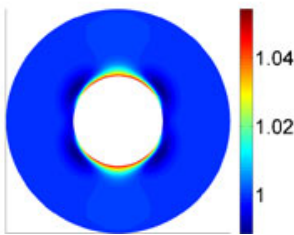

(f) $g(r, R)$ at node 5

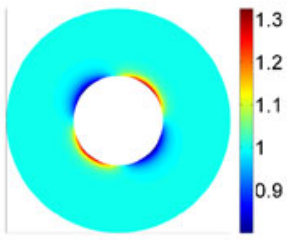

(d) $g(r, R)$ at node 3

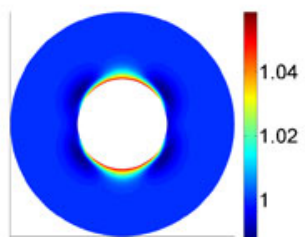

(g) $g(r, R)$ at node 6 setting is a microchannel of $2 \mathrm{H} \times 20 \mathrm{H}$ cross section and 1,040H long with $H=25 \mu \mathrm{m}$. The suspensions are slightly charged hard spheres of diameter $a=1.1 \mu \mathrm{m}$ in a flow characterized by the Péclet number Pe $=69$. For more details concerning the experimental setting, please refer to Frank et al. (2003).
Below, all the geometrical dimensions are given in dimensionless form (by dividing by $H$ which is the half of the height of the channel). The numerical solutions of Eq. 10 are presented for the 2D Poiseuille flow with uncorrelated pair correlation function at the entrance and at the top and bottom walls. Experimental results are fitted by tuning the

Fig. 6 Steady state of $n_{\mathrm{p}}(\boldsymbol{r})$ and $g$ at nodes 1 to 6 with $\kappa^{(\mathrm{I})}=\frac{1}{\boldsymbol{R}^{2}}$
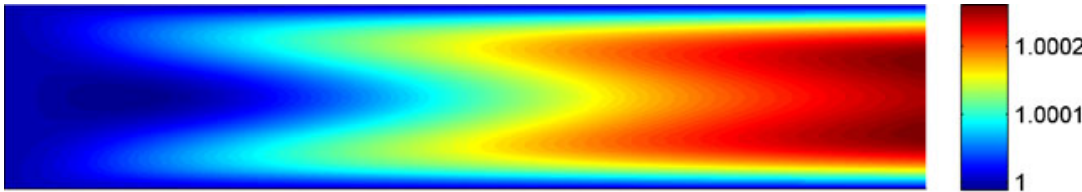

(a) $n_{p}(r)$

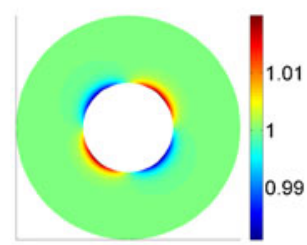

(b) $g(r, R)$ at node 1

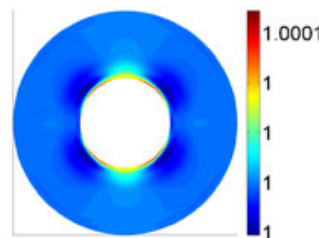

(e) $g(r, R)$ at node 4

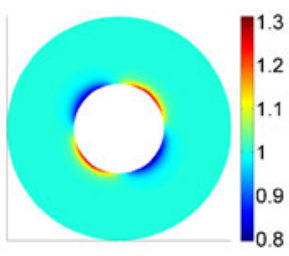

(C) $g(r, R)$ at node 2

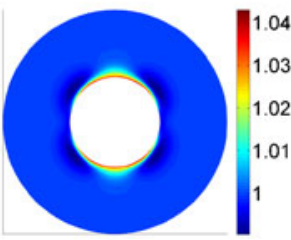

(f) $g(r, R)$ at node 5

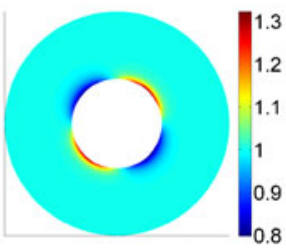

(d) $g(\boldsymbol{r}, \boldsymbol{R})$ at node 3

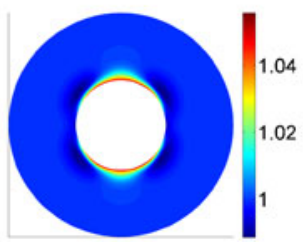

(g) $g(r, R)$ at node 6 
Fig. 7 Steady state of $n_{\mathrm{p}}(\boldsymbol{r})$ and $g$ at nodes 1 to 6 with $\kappa^{(\text {II) }}=\frac{0.005}{\boldsymbol{R}^{2}}$

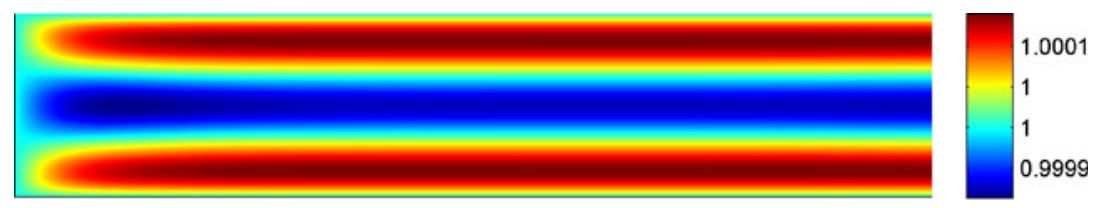

(a) $\mathrm{n}_{\mathrm{p}}(\boldsymbol{r})$

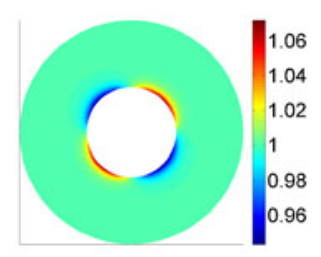

(b) $g(r, R)$ at node 1

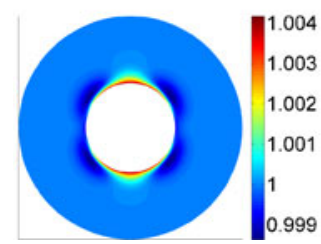

$(\mathrm{e}) g(r, R)$ at node 4

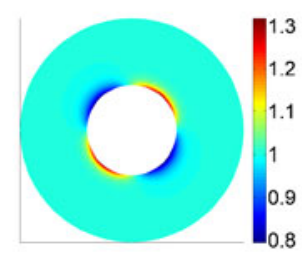

(C) $g(r, R)$ at node 2

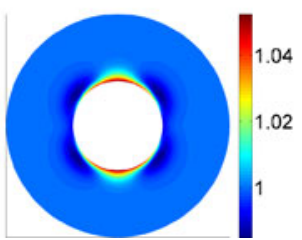

(f) $g(\boldsymbol{r}, \boldsymbol{R})$ at node 5

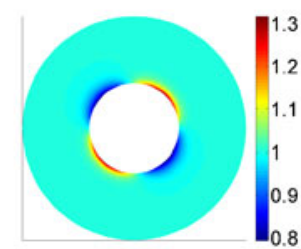

(d) $g(\boldsymbol{r}, \boldsymbol{R})$ at node 3

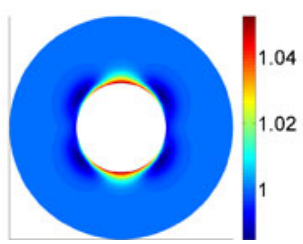

(g) $g(\boldsymbol{r}, \boldsymbol{R})$ at node 6 parameters $\kappa, \kappa^{(\mathrm{I})}, \boldsymbol{\kappa}^{(\mathrm{II})}$, and $\boldsymbol{\kappa}^{(\mathrm{III})}$, which remain for the sake of simplicity in the form presented previously, i.e., $\frac{\alpha}{R^{2}}$. The problem is solved by the PGD method with the same separation as in Eq. 15, and the domains are discretized with 40, 2,100 and 1,680 nodes, respectively, for $x, y$, and $\boldsymbol{R}$-spaces.
Figure 11 shows the normalized local volume fraction of suspended particles as they appear in predictions and in experimental observations. Both types of results appear to be in relatively good agreement.

In order to see more how the parameters $\boldsymbol{\kappa}$ influence predictions of the model, we depict in Fig. 12 the
Fig. 8 Steady state of $n_{\mathrm{p}}(\boldsymbol{r})$ and $g$ at nodes 1 to 6 with $\kappa^{(\text {II) }}=\frac{0.01}{R^{2}}$
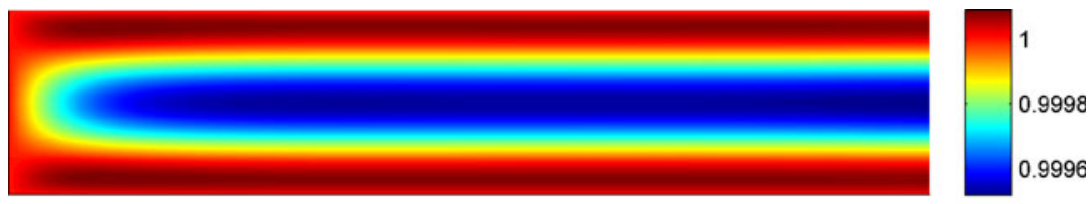

(a) $\mathrm{n}_{\mathrm{p}}(r)$

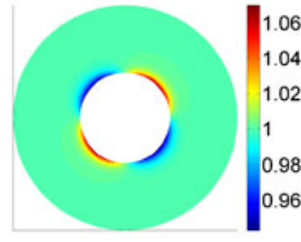

(b) $g(r, R)$ at node 1

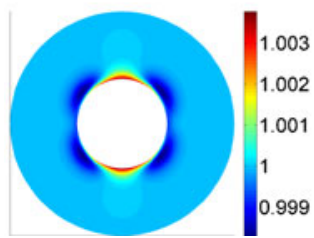

(e) $g(r, R)$ at node 4

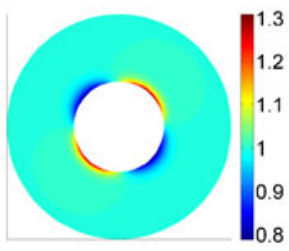

(C) $g(r, R)$ at node 2

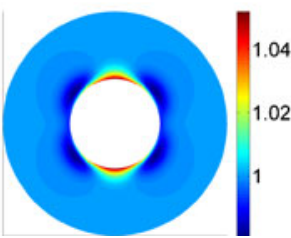

(f) $g(r, R)$ at node 5

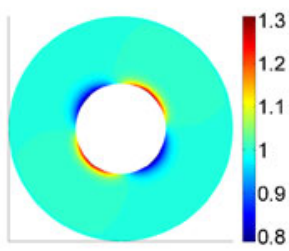

(d) $g(\boldsymbol{r}, \boldsymbol{R})$ at node 3

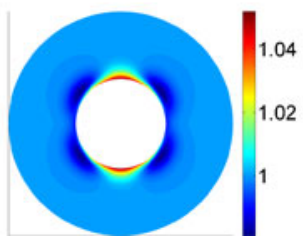

(g) $g(r, R)$ at node 6 
Fig. 9 Steady state of $n_{\mathrm{p}}(\boldsymbol{r})$ and $g$ at nodes 1 to 6 with $\kappa^{(\mathrm{III})}=\frac{0.005}{\boldsymbol{R}^{2}}$

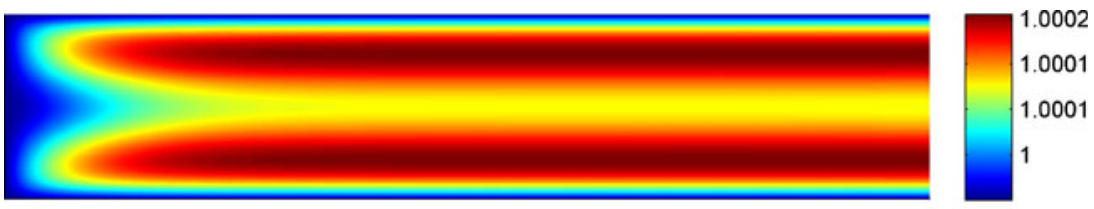

(a) $n_{p}(r)$

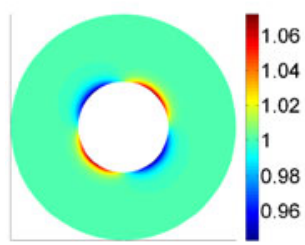

(b) $g(r, R)$ at node 1

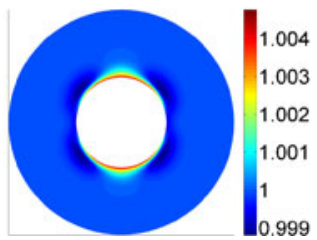

(e) $g(r, R)$ at node 4

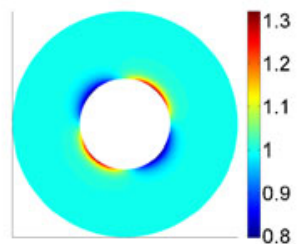

(C) $g(\boldsymbol{r}, \boldsymbol{R})$ at node 2

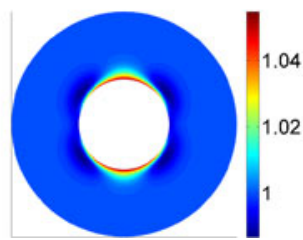

(f) $g(\boldsymbol{r}, \boldsymbol{R})$ at node 5

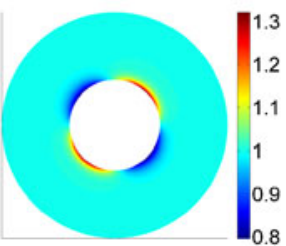

(d) $g(\boldsymbol{r}, \boldsymbol{R})$ at node 3

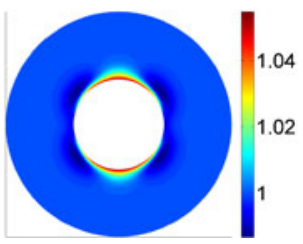

(g) $g(\boldsymbol{r}, \boldsymbol{R}) \alpha \tau \operatorname{vo\delta \varepsilon } 6$ numerically calculated local volume fraction of particle. We can clearly see that the Faxén forces, and in particular the force associated with the parameter $\kappa^{(\mathrm{III})}$, have a strong influence on $\phi$. The diffusion in the $\boldsymbol{r}$-space is kept (i.e., the force proportional to $\boldsymbol{\kappa}$ that drives the diffusion in $\boldsymbol{r}$-space is kept) in order to preserve the numerical stability. In the case when no migration effects are taken into account (neither $\boldsymbol{r}$-space diffusion nor Faxén forces are included), oscillations arising in the solution near the wall (dotted line) emerge. The influence of the other Faxén forces, namely those proportional to $\boldsymbol{\kappa}^{(\mathrm{I})}$ and $\boldsymbol{\kappa}^{(\mathrm{II})}$, is somewhat smaller, and we use them to enhance slightly
Fig. 10 Steady state of $n_{\mathrm{p}}(\boldsymbol{r})$ and $g$ at nodes 1 to 6 with $\kappa^{(\mathrm{III})}=\frac{0.1}{\boldsymbol{R}^{2}}$

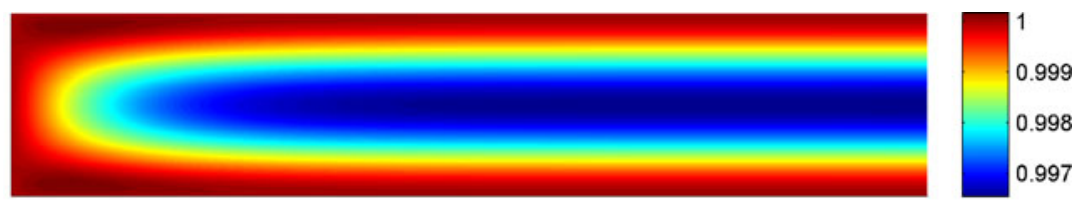

(a) $n_{p}(r)$

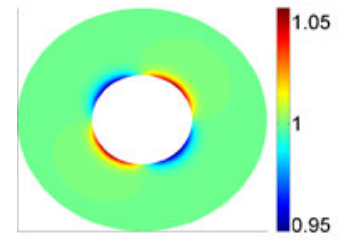

(b) $g(r, R)$ at node 1

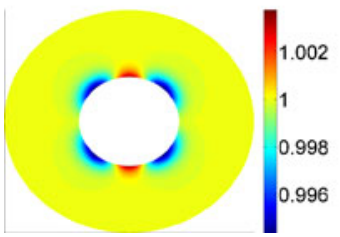

(e) $g(r, R)$ at node 4

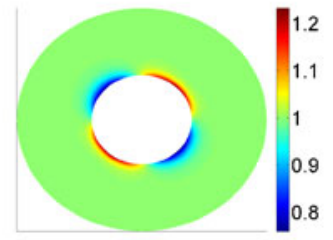

(C) $g(r, R)$ at node 2

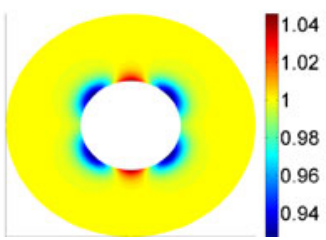

(f) $g(r, R)$ at node 5

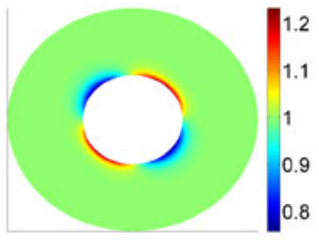

(d) $g(r, R)$ at node 3

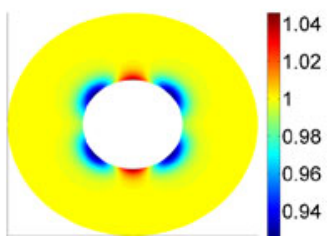

(g) $g(r, R)$ at node 6 
Fig. 11 Normalized local volume fraction of particle for both experimental results (square, from Frank et al. 2003) and modified Smoluchowski model (circle) accounting for migration effects. The parameters used are $\kappa=0.00075 / R^{2}, \kappa^{(\mathrm{I})}=$ $0.05 / R^{2}, \kappa^{(\mathrm{II})}=0.005 / R^{2}$, and $\kappa^{(\text {IIII }}=-0.1 / R^{2}$, and the initial mean volume fraction of particle is $\phi=0.05$

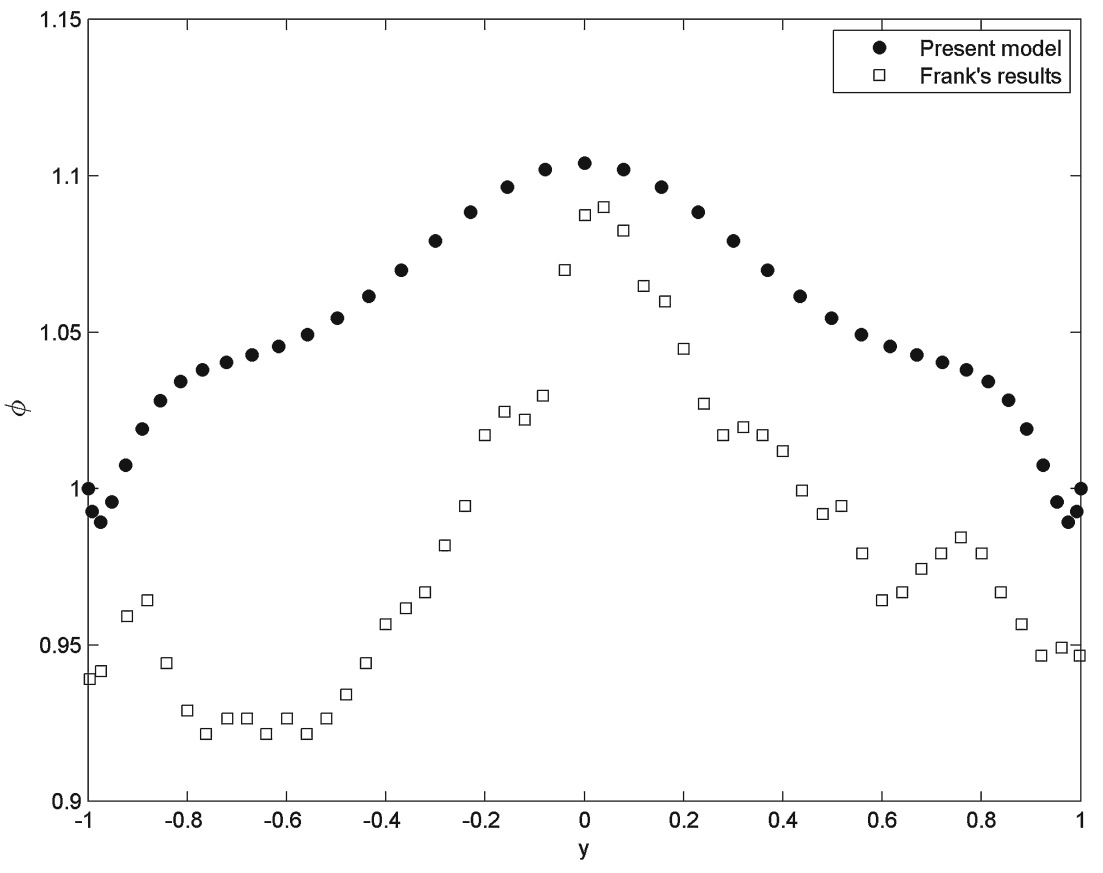

the fitting (especially near the walls where $\phi$ is seen to decrease).

Figure 13 presents $n_{\mathrm{p}}(\boldsymbol{r})$ normalized by its value at rest for the whole geometry. We can indeed observe the migration of the suspended particles toward the center of the channel.

In order to compare the experimental profile with the one predicted by Eq. 1, we reproduce numerically the experiment reported in Semwogerere et al. (2007) (which is very similar to the one presented in Frank et al. 2003).
The flow is characterized by the Péclet number $\mathrm{Pe}=$ 129 , and the suspended particles are slightly charged hard spheres of diameter $a=1.4 \mu \mathrm{m}$. The values chosen for $\kappa, \kappa^{(\mathrm{I})}, \boldsymbol{\kappa}^{(\mathrm{II})}$, and $\kappa^{(\mathrm{III})}$ are $0.00075 / R^{2}, 5 / R^{2}, 0.005 / R^{2}$, and $-0.1 / R^{2}$, respectively. Since the initial mean volume fraction $\phi$ of suspended particles is, in this case, $\phi=$ 0.26 , the kinetic theory of semidilute suspensions discussed in this paper is, strictly speaking, not applicable. Nevertheless, we can see a qualitative agreement (Figs. 14 and 15).
Fig. 12 Influence of the parameters $\kappa, \kappa^{(\mathrm{I})}, \kappa^{(\mathrm{II})}$, and $\kappa^{(\text {III) }}$ on the numerically calculated local volume fraction of particles. The parameter $\kappa \neq 0$ (i.e., a small diffusion in the $\boldsymbol{r}$-space is kept) in order to preserve the numerical stability

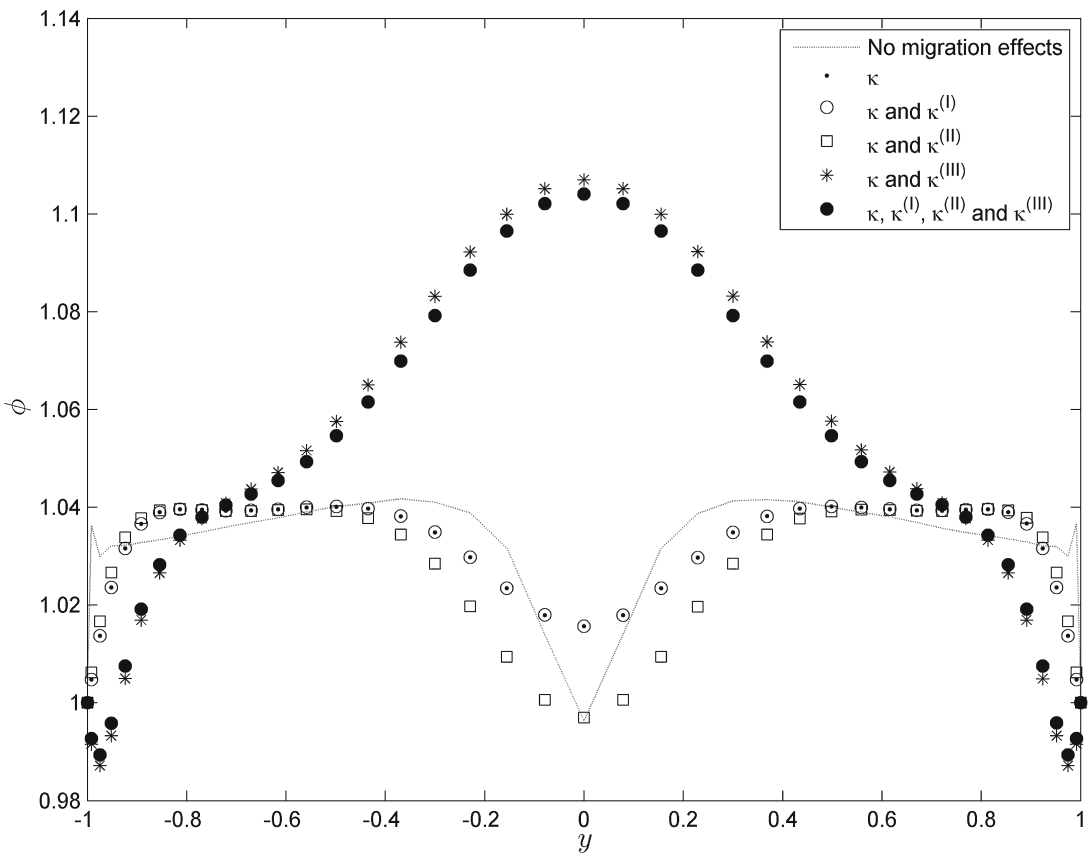


Fig. 13 Normalized $n_{\mathrm{p}}(\boldsymbol{r})$ for the whole channel. Note that for the sake of clarity, different scales are adopted for $x$ - and $y$-axes of the picture

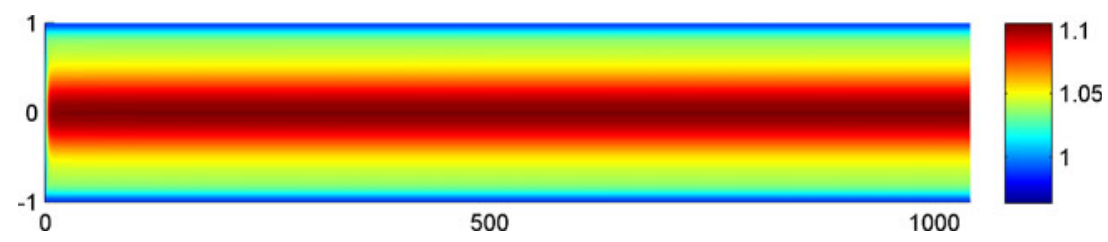

We make several comments about the results presented above:

- In both the experimental and the numerical results, we observe a decrease in the volume fraction of particles near the wall. This means that even if the theoretical boundary condition on the wall (uncorrelated pair correlation function) is likely not completely realistic, it seems to represent at least a good approximation.

- Smoluchowski model supplemented with Faxén forces exhibits also a transition phase between the velocity inlet and the developed microstructure. Thus, with this transition phase, both the numerical profiles at $x / H=80$ and $x / H=1,360$ and the whole channel plot picture, as the experimental ones, were obtained.

- As expected, the magnitude reached in a dense suspension cannot be reproduced by the modified Smoluchowski model.

Summing up, we note that the pair correlation function obtained by solving Eq. 1 is in good agreement with the experimental data, particularly when considering that only a simple form of the parameters $\boldsymbol{\kappa}$ has been used.

Finally, we comment about the numerical calculations. The systems, discretized with 40, 2,700, and 1,680 nodes, have more than 180 millions of degrees of freedom and are solved with the PGD method in less than $1 \mathrm{~h}$.

\section{Concluding remarks}

It has been suggested by Lhuillier (2009) that the forces that play an important role in driving the experimentally observed across-the-streamline particle migration in colloidal suspensions are the Faxén-type forces involving second-order derivatives of the velocity with respect to the position coordinate. In this paper, we follow thisinsight and implement the Faxén forces into theSmoluchowski kinetic equation for the pair correlationfunction and also into the corresponding expression for the stress tensor. With such complete formulation, we are then in the position to investigate both morphology (i.e., the pair correlation function and the migration) and rheology(the stress tensor). The way we derive the kinetic equation is based on the requirement that its solutions are compatible with mechanics and thermodynamics. This approach, called top-down, is explained in the "Introduction" section and in the Appendix. The main results of this paper arethe following:

1. We show that in the context of the top-down approach to kinetic theory, the hydrodynamic interactions that are responsible for creating the flow-induced pair correlations enter as friction forces participating in bringing the relative velocity of two particles to zero and the overall velocity to a uniform velocity.
Fig. 14 Normalized local volume fraction of particle for both experimental results (square, from Semwogerere et al. 2007) and the modified Smoluchowski model (circle) accounting for migration effects. The parameters used in the latter case are $\kappa=0.00075 / R^{2}, \kappa^{(\mathrm{I})}=$ $5 / R^{2}, \kappa^{(\mathrm{II})}=0.005 / R^{2}$, and $\kappa^{\text {(III) }}=-0.1 / R^{2}$, and the initial mean volume fraction of particle is $\phi=0.26$

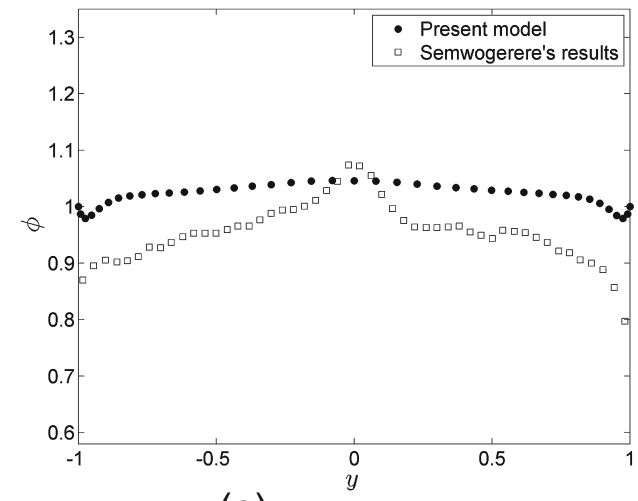

(a) Profile at $\mathrm{H} / \mathrm{x}=80$

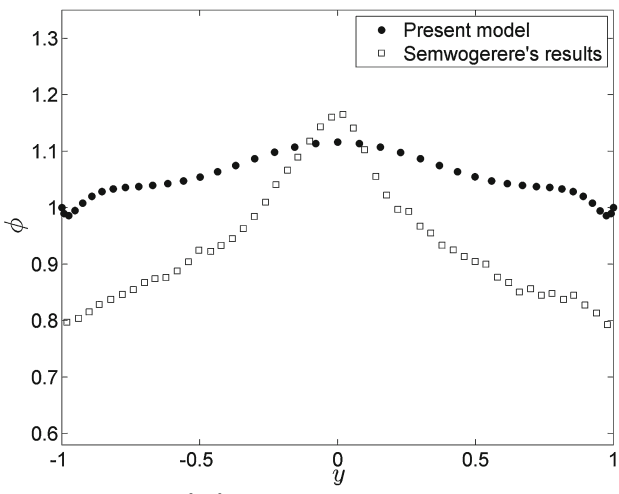

(b) Profile at $H / x=1360$ 
Fig. 15 Normalized $n_{\mathrm{p}}(\boldsymbol{r})$ for the whole channel. Note that for the sake of clarity, different scales are adopted for $x$ - and $y$-axes of the picture

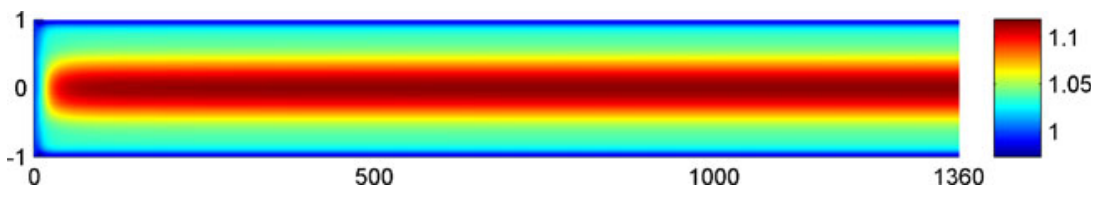

2. We show that in the context of the top-down approach to kinetic theory, the Faxén forces are among the friction forces participating in bringing the velocity of the center of mass of two particles to the overall velocity and the overall velocity to a velocity with uniform gradient.

3. We show, by solving numerically the modified Smoluchowski equation, that the Faxén forces indeed bring about the particle migration observed experimentally.

The numerical solution cannot be found by standard numerical methodssince the unknown function (the pair correlation functionthat we want to find depends on five independent variables in 2D and seven in 3DIt has been demonstrated in Maîtrejean et al. (2012) that the PGD methoddeveloped recently by Ammar et al. (2006, 2007, 2009)and Chinesta et al. (2010, 2011), makes theproblem of finding numerical solutions to the Smoluchowski kinetic equation feasibleAlthough the experimental observations and the microhydrodynamics (Stokesian dynamics) numerical simulations have mainly been donefor rather dense suspensions that lie outside the domain of validity of the modified Smoluchowski kinetic theory developed in this paper,we observe a qualitative agreement between theoreticapredictions and experimental observations.

\section{Appendix: Derivation of Eqs. 1 and 3}

The way the Faxén forces enter the modified Smoluchowski kinetic Eq. (1) and the expression (Eq. 3) for the stress tensor (i.e., the terms involving the parameters $\kappa, \kappa^{(\mathrm{I})}, \boldsymbol{\kappa}^{(\mathrm{II})}$, and $\boldsymbol{\kappa}^{(\mathrm{III})}$ ) can only be explained in the context of their full derivation. We now proceed to do it. The original Smoluchowski kinetic equation has been, of course, derived many times before, but its derivation presented below is different from the previous derivations. It offers a new viewpoint of hydrodynamic interactions, and most importantly, it is directly extendable to the modified Smoluchowski kinetic involving Faxén forces and migration.

We derive Eqs. 1 and 3 by following the top-down method (described in the "Introduction" section) with the mathematical structure in the Hamiltonian form. We now formulate the structure. Let $x$ denote state variables, $\mathcal{E}$ denote energy, and the symbol $\mathcal{S}$, the entropy. Both energy and entropy are real-valued sufficiently regular functions of $x$. By $x^{*}$, we denote conjugate of $x^{*}$ (considered initially as being independent of $x$ but eventually equated to $\mathcal{S}_{x}$ ). The symbol
$\Xi$ stands for another potential, called a dissipation potential, that is a real-valued sufficiently regular function of $x$ and $x^{*}$. The equation governing the time evolution of $x$ is given by

$\frac{\partial x}{\partial t}=L(x) \mathcal{E}_{x}+\left.\Xi\left(x, x^{*}\right)_{x^{*}}\right|_{x^{*}=\mathcal{S}_{x}}$.

By $\mathcal{E}_{x}$, we denote a derivative of $\mathcal{E}$ with respect to $x$; similarly, $\mathcal{S}_{x}$ denotes a derivative of $\mathcal{S}$ with respect to $x$ and $\Xi\left(x, x^{*}\right)_{x^{*}}$, a derivative of $\Xi$ with respect to $x^{*}$. The symbol $\left.\right|_{x^{*}=\mathcal{S}_{x}}$ denotes that the expression appearing on the left of $\mid$ is evaluated at $x^{*}=\mathcal{S}_{x}$. The symbol $L(x)$ denotes an operator, depending in general on $x$, that transforms a covector into a vector. Both the dissipation potential $\Xi$ and the operator $L$ are required to satisfy certain properties that will be listed below in the particular context of the time evolution discussed in this paper (for a general presentation, see Grmela 2010a). The abstract Eq. 16 has arisen gradually in Dzyaloshinskii and Volovick (1980), Grmela (1984a, b), Kaufman (1984), Morrison (1984), Beris and Edwards (1994), Grmela and Öttinger (1997), and Öttinger and Grmela (1997). It has been called GENERIC in Grmela and Öttinger (1997) and Öttinger and Grmela (1997). An investigation of Eq. 16 leading to a further illumination of its physical content and to extensions represents a very active domain of research (see, e.g., Grmela 2010a, 2012a where its thermodynamic content is explored and Grmela 2005, 2010 where its quantum mechanical version is developed). Below, we shall use it in the form appearing in Grmela and Öttinger (1997).

As for the physical content of Eq. 16, we recall (see the "Introduction" section and references cited therein) that solutions to Eq. 16 are guaranteed to be compatible with mechanics and thermodynamics. The first term on the righthand side of Eq. 16 expresses the compatibility with mechanics. Indeed, we recall that Newton's equations, if written in the Hamiltonian form, are $\frac{d x}{d t}=L(x) \mathcal{E}_{x}$, where $x=\left(\begin{array}{l}r \\ v\end{array}\right)$ and $L=\left(\begin{array}{cc}0 & 1 \\ -1 & 0\end{array}\right) ; v$ is the momentum and $r$ position coordinate. We recall that $\left(a_{r}, a_{v}\right)\left(\begin{array}{cc}0 & 1 \\ -1 & 0\end{array}\right)\left(\begin{array}{l}b_{r} \\ b_{v}\end{array}\right)$ is the classical canonical Poisson bracket ( $a$ and $b$ are real-valued sufficiently general functions of $r$ and $v$ ), and we thus require that $\left\langle a_{x}, L b_{x}\right\rangle$, where $a$ and $b$ are real-valued sufficiently regular functions of $x$ and $<,>$ denotes the scalar product, has all the properties required from the Poisson bracket (denoted hereafter by the symbol $\{a, b\}$ ). The 
operator $L(x)$ and the its associated Poisson bracket can be physically interpreted as quantities kinematics of $x$ is expressed. The second term on the right-hand side of Eq. 16 addresses the compatibility with thermodynamics. It makes the entropy $\mathcal{S}(x)$ to increase in time and guarantees the approach of $x$ to equilibrium states. Due to our limitation to isothermal suspensions, Eq. 16 can be immediately simplified. Instead of two potentials, namely the energy $\mathcal{E}$ and the entropy $\mathcal{S}$, we need only one potential $\Phi=\mathcal{E}-k_{\mathrm{B}} T \mathcal{S}$, called a free energy; $k_{\mathrm{B}}$ is the Boltzmann constant and $T$ is a constant temperature. Equation 16 takes, for the isothermal systems, the form

$\frac{\partial x}{\partial t}=L \Phi_{x}-\left.\Xi\left(x, x^{*}\right)_{x^{*}}\right|_{x^{*}=\Phi_{x}}$.

Our objective now is to construct a particular realization of Eq. 17 in which the physics of suspensions under consideration is expressed. We recognize in Eq. 17 four modules: the state variables $x$, their Hamiltonian kinematics $L(x)$, the dissipation potential $\Xi\left(x, x^{*}\right)$, and the energy $\Phi(x)$. By a particular realization of Eq. 16, we mean Eq. 16 in which all the four modules acquire a concrete form. Below, we shall make the specifications.

State variables $x$

As we have already recalled in the "Introduction" section, the morphology of suspensions of rigid spheres is expressed in the pair correlation function. Such function, denoted by $g(\boldsymbol{r}, \boldsymbol{R})$ (see the text below, Eq. 1 has to be thus a part of fields included in state variables. The remaining two fields that we shall include are $\rho(\boldsymbol{r})$ and $\boldsymbol{u}(\boldsymbol{r})$, which denote the overall mass density and the overall momentum of the suspension. The complete set of state variables that we suggest is thus

$x=(\rho(\boldsymbol{r}), \boldsymbol{u}(\boldsymbol{r}), g(\boldsymbol{r}, \boldsymbol{R}))$.

These are indeed the fields whose time evolution is governed by the kinetic Eq. 1 and the standard fluid mechanics equations $\rho$ and $\boldsymbol{u}$, with the stress tensor given by Eq. 3 .

In the investigation of the other modules, in particular already in the investigation of the next modulus that addresses kinematics, it turns out that it is convenient to start with another set of state variables, namely with

$x=(\rho(\boldsymbol{r}), \boldsymbol{u}(\boldsymbol{r}), g(\boldsymbol{r}, \boldsymbol{R}), \boldsymbol{U}(\boldsymbol{r}, \boldsymbol{R}), \boldsymbol{W}(\boldsymbol{r}, \boldsymbol{R}))$.

The two extra fields $\boldsymbol{U}(\boldsymbol{r}, \boldsymbol{R})$ and $\boldsymbol{W}(\boldsymbol{r}, \boldsymbol{R}))$ denote the momentum and the relative momentum of the two particles, respectively. Only at the end (see the "Reduction from the state variables (19) to the state variables (18)" section), when we have already written down a complete set of the time evolution equations for Eq. 19, we eliminate the fields $\boldsymbol{U}$ and $\boldsymbol{W}$ by assuming that the material parameters in the equations are chosen in such a way that the fields $\boldsymbol{U}$ and $\boldsymbol{W}$ evolve more rapidly than the fields (18) and by restricting ourselves to the stage in the time evolution in which $\boldsymbol{U}$ and $\boldsymbol{W}$ become completely enslaved to the fields (18).

We end this subsection by relating the fields $(g(\boldsymbol{r}, \boldsymbol{R}), \boldsymbol{U}(\boldsymbol{r}, \boldsymbol{R}), \boldsymbol{W}(\boldsymbol{r}, \boldsymbol{R}))$ to the two-particle correlation function $\psi\left(\boldsymbol{r}_{1}, \boldsymbol{v}_{1}, \boldsymbol{r}_{2}, \boldsymbol{v}_{2}\right)$, where $\left(\boldsymbol{r}_{1}, \boldsymbol{r}_{2}\right)$ and $\left.\boldsymbol{v}_{1}, \boldsymbol{v}_{2}\right)$ are position vectors and momenta of the two particles, respectively. First, we introduce the new coordinates

$$
\begin{array}{ll}
r & =\frac{1}{2}\left(\boldsymbol{r}_{1}+\boldsymbol{r}_{2}\right) ; \quad \boldsymbol{R}=\boldsymbol{r}_{2}-\boldsymbol{r}_{1} ; \\
\boldsymbol{v} & =\boldsymbol{v}_{1}+\boldsymbol{v}_{2} ; \quad \boldsymbol{V}=\frac{1}{2}\left(\boldsymbol{v}_{2}-\boldsymbol{v}_{1}\right)
\end{array}
$$

which transform $\psi\left(\boldsymbol{r}_{1}, \boldsymbol{v}_{1}, \boldsymbol{r}_{2}, \boldsymbol{v}_{2}\right)$ into $\psi(\boldsymbol{r}, \boldsymbol{v}, \boldsymbol{R}, \boldsymbol{V})$ (note that the Jacobian of the transformation (20) equals 1) and then introduce

$$
\begin{aligned}
g(\boldsymbol{r}, \boldsymbol{R}) & =\int d \boldsymbol{v} \int \boldsymbol{d} \boldsymbol{V} \psi(\boldsymbol{r}, \boldsymbol{v}, \boldsymbol{R}, \boldsymbol{V}) \\
U_{\alpha}(\boldsymbol{r}, \boldsymbol{R}) & =\int d \boldsymbol{v} \int \boldsymbol{d} \boldsymbol{V} V_{\alpha} \psi(\boldsymbol{r}, \boldsymbol{v}, \boldsymbol{R}, \boldsymbol{V}) \\
W_{\alpha}(\boldsymbol{r}, \boldsymbol{R}) & =\int d \boldsymbol{v} \int d \boldsymbol{V} v_{\alpha} \psi(\boldsymbol{r}, \boldsymbol{v}, \boldsymbol{R}, \boldsymbol{V}) .
\end{aligned}
$$

Kinematics L

The Poisson bracket expressing the kinematics of the state variables (19) is given by

$\{a, b\}=\{a, b\}^{(\text {hyd })}+\{a, b\}^{(g, U, W)}+\{a, b\}^{(h y d, g, U, W)}$

where

$$
\begin{aligned}
& \{a, b\}^{\text {(hyd) }}=\int d \boldsymbol{r} \rho\left(\frac{\partial a_{\rho}}{\partial r_{i}} b_{u_{i}}-\frac{\partial b_{\rho}}{\partial r_{i}} a_{u_{i}}\right) \\
& +\int d \boldsymbol{r} u_{j}\left(\frac{\partial a_{u_{j}}}{\partial r_{i}} b_{u_{i}}-\frac{\partial b_{u_{j}}}{\partial r_{i}} a_{u_{i}}\right), \\
& \{a, b\}^{(g, U, W)}=\int d \boldsymbol{r} \int d \boldsymbol{R}\left[g\left(\frac{\partial a_{g}}{\partial r_{j}} b_{W_{j}}-\frac{\partial b_{g}}{\partial r_{j}} a_{W_{j}}\right)\right. \\
& +U_{k}\left(\frac{\partial a_{U_{k}}}{\partial r_{j}} b_{W_{j}}-\frac{\partial b_{U_{k}}}{\partial r_{j}} a_{W_{j}}\right) \\
& +W_{k}\left(\frac{\partial a_{W_{k}}}{\partial r_{j}} b_{W_{j}}-\frac{\partial b_{W_{k}}}{\partial r_{j}} a_{W_{j}}\right) \\
& +g\left(\frac{\partial a_{g}}{\partial R_{j}} b_{U_{j}}-\frac{\partial b_{g}}{\partial R_{j}} a_{U_{j}}\right) \\
& +U_{k}\left(\frac{\partial a_{U_{k}}}{\partial R_{j}} b_{U_{j}}-\frac{\partial b_{U_{k}}}{\partial R_{j}} a_{U_{j}}\right) \\
& \left.+W_{k}\left(\frac{\partial a_{W_{k}}}{\partial R_{j}} b_{U_{j}}-\frac{\partial b_{W_{k}}}{\partial R_{j}} a_{U_{j}}\right)\right] \text {, }
\end{aligned}
$$


and

$\{a, b\}^{(h y d, g, U, W)}$

$$
\begin{aligned}
=\int d \boldsymbol{r} \int d \boldsymbol{R}[ & 2 g\left(\frac{\partial a_{\rho}}{\partial r_{j}} b_{W_{j}}-\frac{\partial b_{\rho}}{\partial r_{j}} a_{W_{j}}\right) \\
& +g\left(\frac{\partial a_{g}}{\partial r_{j}} b_{u_{j}}-\frac{\partial b_{g}}{\partial r_{j}} a_{u_{j}}\right) \\
& +U_{k}\left(\frac{\partial a_{U_{k}}}{\partial r_{j}} b_{u_{j}}-\frac{\partial b_{U_{k}}}{\partial r_{j}} a_{u_{j}}\right) \\
& +W_{k}\left(\frac{\partial a_{W_{k}}}{\partial r_{j}} b_{u_{j}}-\frac{\partial b_{W_{k}}}{\partial r_{j}} a_{u_{j}}\right) \\
& +g R_{l}\left(\frac{\partial a_{g}}{\partial R_{j}} \frac{\partial b_{u_{j}}}{\partial r_{l}}-\frac{\partial b_{g}}{\partial R_{j}} \frac{\partial a_{u_{j}}}{\partial r_{l}}\right) \\
& +R_{l} U_{k}\left(\frac{\partial a_{U_{k}}}{\partial R_{j}} \frac{\partial b_{u_{j}}}{\partial r_{l}}-\frac{\partial b_{U_{k}}}{\partial R_{j}} \frac{\partial a_{u_{j}}}{\partial r_{l}}\right) \\
& +R_{l} W_{k}\left(\frac{\partial a_{W_{k}}}{\partial R_{j}} \frac{\partial b_{u_{j}}}{\partial r_{l}}-\frac{\partial b_{W_{k}}}{\partial R_{j}} \frac{\partial a_{u_{j}}}{\partial r_{l}}\right) \\
& -U_{l}\left(a_{U_{i}} \frac{\partial b_{u_{l}}}{\partial r_{i}}-b_{U_{i}} \frac{\partial a_{u_{l}}}{\partial r_{i}}\right) \\
& \left.-W_{l}\left(a_{W_{i}} \frac{\partial b_{u_{l}}}{\partial r_{i}}-b_{W_{i}} \frac{\partial a_{u_{l}}}{\partial r_{i}}\right)\right] .
\end{aligned}
$$

With this Poisson bracket, Eq. 17 without the second term on its right-hand side takes the form (by ( $)_{\text {rev }}$ we denote the reversible and nondissipative part of the time evolutioni.e., the time evolution governed by Eq. 17 without the second term on its right-hand side)

$$
\begin{aligned}
\left(\frac{\partial \rho}{\partial t}\right)_{\mathrm{rev}}= & -\frac{\partial\left(\rho \Phi_{u_{j}}\right)}{\partial r_{j}}-2 \frac{\partial\left(\int d \boldsymbol{R}_{g} \Phi_{W_{j}}\right)}{\partial r_{j}} \\
\left(\frac{\partial u_{i}}{\partial t}\right)_{\mathrm{rev}}= & -\frac{\partial\left(u_{i} \Phi_{u_{j}}\right)}{\partial r_{j}}-\frac{\partial p}{\partial r_{i}}-\frac{\partial \sigma_{i j}}{\partial r_{j}} \\
\left(\frac{\partial g}{\partial t}\right)_{\mathrm{rev}}= & -\frac{\partial\left(g \Phi_{u_{j}}\right)}{\partial r_{j}}-\frac{\partial\left(g \Phi_{W_{j}}\right)}{\partial r_{j}} \\
\left(\frac{\partial U_{i}}{\partial t}\right)_{\mathrm{rev}}= & -\frac{\partial\left(g \Phi_{U_{j}}\right)}{\partial R_{j}}-\frac{\partial\left(g R_{l} \frac{\partial \Phi_{u_{j}}}{\partial r_{l}}\right)}{\left.\partial \Phi_{u_{j}}\right)}-\frac{\partial\left(U_{i} \Phi_{W_{j}}\right)}{\partial r_{j}} \\
& -\frac{\partial\left(U_{i} \Phi_{U_{j}}\right)}{\partial R_{j}}-\frac{\partial\left(R_{l} U_{i} \frac{\partial \Phi_{u_{j}}}{\partial r_{l}}\right)}{\partial R_{j}} \\
& -g \frac{\partial \Phi_{g}}{\partial R_{i}}-U_{l} \frac{\partial \Phi_{u_{l}}}{\partial r_{i}}-U_{l} \frac{\partial \Phi_{U_{l}}}{\partial R_{i}}-W_{l} \frac{\partial \Phi_{W_{l}}}{\partial R_{i}} \\
& -\frac{\partial\left(W_{i} \Phi_{u_{j}}\right)}{\partial r_{j}}-\frac{\partial\left(W_{i} \Phi_{W_{j}}\right)}{\partial r_{j}}-\frac{\partial\left(W_{i} \Phi_{U_{j}}\right)}{\partial R_{j}} \\
\left(\frac{\partial W_{i}}{\partial t}\right)_{\mathrm{rev}}= & \frac{\partial\left(R_{l} W_{i} \frac{\partial \Phi_{u_{j}}}{\partial r_{l}}\right)}{\partial R_{j}}-U_{l} \frac{\partial \Phi_{U_{l}}}{\partial r_{i}}-W_{l} \frac{\partial \Phi_{W_{l}}}{\partial r_{i}} \\
& -\frac{\partial \Phi_{\rho}}{\partial r_{i}} \\
& \left.-\frac{\partial r_{i}}{\partial}\right)
\end{aligned}
$$

where $p(\boldsymbol{r})$, the scalar hydrostatic pressure, is given by

$p=-\varphi+\rho \Phi_{\rho}+u_{j} \Phi_{u_{j}}+\int d \boldsymbol{R}\left[g \Phi_{g}+U_{j} \Phi_{U_{j}}+W_{j} \Phi_{W_{j}}\right]$.

$\varphi(\boldsymbol{r})$ is the density of the free energy (i.e., $\Phi=\int d \boldsymbol{r} \varphi(\boldsymbol{r})$ ), and the stress tensor $\boldsymbol{\sigma}(\boldsymbol{r})$ is given by

$$
\begin{aligned}
\sigma_{i j}= & -\int d \boldsymbol{R}\left[g R_{j} \frac{\partial \Phi_{g}}{\partial R_{i}}+R_{j} U_{k} \frac{\partial \Phi_{U_{k}}}{\partial R_{i}}+R_{j} W_{k} \frac{\partial \Phi_{E_{k}}}{\partial R_{i}}\right. \\
& \left.-U_{i} \Phi_{U_{j}}-W_{i} \Phi_{W_{j}}\right] .
\end{aligned}
$$

Two questions remain to be answered.

Question 1 How did we arrive at Eq. 22 and what is the physics behind it?

and

\section{Question 2 Is (22) indeed a Poisson bracket?}

As for the first question, we can answer it in two ways. First, we just accept it as a guess and look for the answer in its consequences. For instance, we shall see below that with this bracket, we arrive at the classical Smoluchowski equation that has been derived previously in the bottom-up way from microhydrodynamics. This constitutes indeed an indirect proof of the physical significance of Eq. 22. The second way to answer the first question is to provide its bottom-up derivation.

In the rest of this subsection, we shall first answer the second question (see the "Mathematical aspects of the bracket (22)" section) and then interpret the mathematical proof as the bottom-up derivation of Eq. 22 (see the "Physical aspects of the bracket (22)" section).

\section{Mathematical aspects of the bracket (22)}

We recall that Eq. 22 is a Poisson bracket if $\{a, b\}=-\{b, a\}$ and the Jacobi identity $\{\{a, b\}, c\}+\{\{b, c\}, a\}+\{\{c, a\}, b\}=$ 0 holds. While the first property is manifestly visible in Eq. 22, the second property is more difficult to prove. The proof can be made either directly (a computer-assisted way to verify the Jacobi identity for brackets of the type Eq. 22 is described in Kröger and Hütter (2010)) or indirectly. We shall take the latter route.

First, we present two lemmas that we shall use in the proof.

Lemma (A) Let $a$ and $b$ be sufficiently regular real-valued functions of $x$ and $\{a(x), b(x)\}$ a Poisson bracket. Let $x \leftrightarrow$ $y$ be a one-to-one and sufficiently regular transformation. Then, $\{a(y), b(y)\}$ is also a Poisson bracket. 
Proof This property is, of course, essential for the role that the Poisson bracket plays in geometry.

Lemma (B) Let $a$ and $b$ be sufficiently regular real-valued functions of $x$ and let $\mathcal{D}$ be a set of all such functions. Let $\{a(x), b(x)\}$ be a Poisson bracket. By $\widetilde{a}$ and $\widetilde{b}$, we denote functions belonging to a subset $\mathcal{C} \subset \mathcal{D}$. If the bracket $[\{a(x), b(x)\}]_{\mathcal{C}}$ (i.e., the bracket $\{a(x), b(x)\}$ in which $a$ and $b$ are restricted to only those that lie in $\mathcal{C})$ involves only functions belonging to $\mathcal{C}$, then $[\{a(x), b(x)\}]_{\mathcal{C}}$ is a Poisson bracket.

Proof The proof is obvious. In most applications of this lemma, $x$ is a function, say $f(\boldsymbol{r})$, and the subset $\mathcal{C}$ is composed of functions $a(f)$ that depend on $f$ only through their dependence on selected moments of $f$ (e.g., on the moment $\left.a_{i j}=\int d \boldsymbol{r} r_{i} r_{j} f(\boldsymbol{r})\right)$.

Now, we begin to prove that Eq. 22 is a Poisson bracket. As the point of departure, we take

$x=\left(f_{1}(1), f_{2}(1,2)\right)$.

We use the shorthand notations $(1)=\left(\boldsymbol{r}_{1}, \boldsymbol{v}_{1}\right)$ and $(1,2)=$ $\left(\boldsymbol{r}_{1}, \boldsymbol{v}_{1}, \boldsymbol{r}_{2}, \boldsymbol{v}_{2}\right)$, where $\boldsymbol{r}_{1}, \boldsymbol{r}_{2}$ and $\boldsymbol{v}_{1}, \boldsymbol{v}_{2}$ are position coordinates of particles 1 and 2 and their momenta, respectively. By $f_{1}(1)$, we denote a 6-form (the volume element) $f_{1} d 1$, and similarly, $f_{2}(1,2)$ is a 12 -form $f_{2} d 1 d 2$.

The Poisson bracket expressing the kinematics of Eq. 29 is given by

$\{a, b\}^{(\mathrm{I})}=\{a, b\}^{(1)}+\{a, b\}^{(2)}$

where

$\{a, b\}^{(1)}=\int d 1\left(\frac{\partial a}{\partial r_{1, i}} \frac{\partial b}{\partial v_{1, i}}-\frac{\partial b}{\partial r_{1, i}} \frac{\partial a}{\partial v_{1, i}}\right)$

and

$$
\begin{aligned}
\{a, b\}^{(2)}= & \int d 1 \int d 2\left(\frac{\partial a}{\partial r_{1, i}} \frac{\partial b}{\partial v_{1, i}}-\frac{\partial b}{\partial r_{1, i}} \frac{\partial a}{\partial v_{1, i}}\right) \\
& +\int d 1 \int d 2\left(\frac{\partial a}{\partial r_{2, i}} \frac{\partial b}{\partial v_{2, i}}-\frac{\partial b}{\partial r_{2, i}} \frac{\partial a}{\partial v_{2, i}}\right) .
\end{aligned}
$$

The brackets Eqs. 31 and 32 are Poisson brackets (they are the Poisson brackets arising in the Liouville representation of classical Poisson brackets of particle mechanics-see Arnold 1989; Marsden and Ratiu 1999), and thus, also Eq. 30 is a Poisson bracket.

As the first step in the passage from Eq. 30 to Eq. 22, we introduce the transformation

$\left(f_{1}(1), f_{2}(1,2)\right) \leftrightarrow\left(\phi_{1}(1), \phi_{2}(1,2)\right)$

$\phi_{1}(1)=f_{1}(1)+\int d 2 \int d 3 f_{2}(2,3)[\delta(1-3)+\delta(1-2)]$,

$\phi_{2}(1,2)=f_{2}(1,2)$.
The transformation (33) is clearly one to one, and thus under this transformation, the Poisson bracket $\{a, b\}^{(\mathrm{I})}$ transforms (by virtue of Lemma (A)) into another Poisson bracket that we shall denote $\{a, b\}^{(\mathrm{II})}$. The calculations leading from $\{a, b\}^{(\mathrm{I})}$ to $\{a, b\}^{(\mathrm{II})}$ are routine, and we shall omit them as well as the explicit form of $\{a, b\}^{(\mathrm{II})}$.

In the second step, we introduce another transformation:

$\left(\phi_{1}(1), \phi_{2}(1,2)\right) \rightarrow(\rho(\boldsymbol{r}), \boldsymbol{u}(\boldsymbol{r}), \psi(1,2))$

$$
\begin{aligned}
\rho(\boldsymbol{r}) & =\int d 1 \phi_{1}(1) \delta\left(\boldsymbol{r}_{1}-\boldsymbol{r}\right), \\
u_{i}(\boldsymbol{r}) & =\int d 1 v_{i} \phi_{1}(1) \delta\left(\boldsymbol{r}_{1}-\boldsymbol{r}\right) . \\
\psi(1,2) & =\phi_{2}(1,2) .
\end{aligned}
$$

The transformation (35) is not one to one, but it turns out that if we restrict the functions $a$ and $b$ in $\{a, b\}^{(\mathrm{II})}$ to those that depend on $\left(\phi_{1}(1), \phi_{2}(1,2)\right)$ only through their dependence on $(\rho(\boldsymbol{r}), \boldsymbol{u}(\boldsymbol{r}), \psi(1,2))$ (the subset of functions of this type forms the subset $\mathcal{C}$ in Lemma (B)), then we obtain a bracket that we shall denote $\{a, b\}^{(\mathrm{III})}$, involving only the functions in $\mathcal{C}$. The bracket $\{a, b\}^{(\mathrm{III})}$ is thus, by virtue of Lemma (B), a Poisson bracket.

In the third step, we introduce the transformation

$(\rho(\boldsymbol{r}), \boldsymbol{u}(\boldsymbol{r}), \psi(1,2)) \leftrightarrow(\rho(\boldsymbol{r}), \boldsymbol{u}(\boldsymbol{r}), \psi(\boldsymbol{r}, \boldsymbol{v}, \boldsymbol{R}, \boldsymbol{V}))$

where $\left(\boldsymbol{r}_{1}, \boldsymbol{v}_{1}, \boldsymbol{r}_{2}, \boldsymbol{v}_{2}\right)$ are related to $(\boldsymbol{r}, \boldsymbol{v}, \boldsymbol{R}, \boldsymbol{V})$ by Eq. 20. The transformation (37) is clearly one to one, and thus, by virtue of Lemma (A), the Poisson bracket $\{a, b\}^{(\mathrm{III})}$ transforms into another Poisson bracket that we shall denote $\{a, b\}^{(\mathrm{IV})}$

Finally, we make the fourth step in which we make the transformation (21)

$$
\begin{array}{r}
(\rho(\boldsymbol{r}), \boldsymbol{u}(\boldsymbol{r}), \psi(\boldsymbol{r}, \boldsymbol{v}, \boldsymbol{R}, \boldsymbol{V})) \rightarrow(\rho(\boldsymbol{r}), \boldsymbol{u}(\boldsymbol{r}), g(\boldsymbol{r}, \boldsymbol{R}), \\
\boldsymbol{U}(\boldsymbol{r}, \boldsymbol{R}), \boldsymbol{W}(\boldsymbol{r}, \boldsymbol{R})) .
\end{array}
$$

This transformation is not one to one, but as in the second step, by virtue of Lemma (B), we arrive at the Poisson bracket (22).

This concludes the proof.

\section{Physical aspects of the bracket (22)}

As we have already suggested in the text following (28), we can see the physics behind (22) (about which we know now that it is indeed a Poisson bracket) in its consequences. In particular, we can see it in the fact that Eq. 22 together with an appropriately chosen dissipation potential (discussed below in the "Dissipation potential $\Xi$ " section) implies the classical Smoluchowski kinetic equation whose microhydrodynamic background is well known and well established on the bottom-up path. 
But we can also see the physics behind (22) by reinterpreting the mathematical analysis presented in the "Mathematical aspects of the bracket (22)" section above as its bottom-up derivation. We now proceed to provide such interpretation.

We begin on the microscopic level of description on which we use particle distribution functions as state variables; $f_{1}$ denotes a one-particle distribution function and $f_{2}$, a two-particle distribution function. At this point, we are not introducing any restrictions (as, for example, a normalization) on these functions.

The physical interpretation of the transformations (35), (37), and (38) is clear. We are just replacing, following the standard procedure developed in kinetic theory, the distribution functions with first two moments that represent the mass and momentum fields.

The most important and the key transformation is Eq. 33. Its main purpose is to introduce a description with two types of state variables: one characterizing overall macroscopic features of the suspension (characterized by the overall momentum field $\boldsymbol{u}(\boldsymbol{r}))$ and the other, an additional microstructure (characterized by the particle pair correlation function $g(\boldsymbol{r}, \boldsymbol{R}))$. At this point, we are only concentrating on the kinematics of the state variables. Constraints placed on them, as for example various normalizations (if distribution functions are among the state variables) or realvalued functions of the state variables with required physical meanings, will be discussed later.

We begin with two descriptions: one in which $f_{1}$ serves as the state variable and the other in which $f_{2}$ plays the same role. These two levels of description, denoted by $\mathcal{L}_{1}$ and $\mathcal{L}_{2}$, respectively, are completely independent. The level $\mathcal{L}_{2}$ takes into account more details (is more microscopic) than the level $\mathcal{L}_{1}$. On both levels, we know the Poisson brackets expressing the kinematics. We now consider our system under investigation simultaneously from both levels of description (we construct a new level of description $\mathcal{L}_{1 \times 2}$ ). If we use the state variables (29) on $\mathcal{L}_{1 \times 2}$, then the combined description is simply a superposition of two independent viewpoints. The transformation (33) mixes the viewpoints. The one-particle distribution function $\phi_{1}$ represents now the less detailed description but with an additional information coming from the original level $\mathcal{L}_{2}$. It characterizes the overall macroscopic behavior in $\mathcal{L}_{1 \times 2}$. The two-particle distribution function $\phi_{2}$ provides only the extra information about the system that is impossible to see with only the one-particle distribution function. Since the transformation (33) is one to one, the Poisson bracket (30) representing the kinematics in $\mathcal{L}_{1 \times 2}$ transforms (by Lemma (A)) into a Poisson bracket representing the kinematics of $\left(\phi_{1}, \phi_{2}\right)$.

The physical interpretation of $\left(\phi_{1}, \phi_{2}\right)$ is further specified by specifying potentials (i.e., real-valued functions of the distribution functions) with specific physical interpretations (mass, number density of suspended particles, energy, and entropy (see Eq. 2) and the potentials introduced in the "Free energy" section).

To the best of our knowledge, the first example of coupling different levels of description that appeared in statistical mechanics is that of Green's interpretation of the grand canonical ensemble in equilibrium statistical mechanics (see Green and Jancovici 1966). In this analysis, the starting point is an infinite number of levels of descriptions that are at first completely independent. These levels differ by the number of particles composing the macroscopic system under considerations. The macroscopic system is the same in all levels, the only difference is in the number of particles in it. The point of departure is the state variable $x=\left(f_{0}, f_{1}, f_{2}, \ldots\right)$, where $f_{0}, f_{1}, f_{2}, \ldots$ denote the distribution functions of the system composed of $0,1,2, \ldots$ number of particles, respectively. The next step in Green's analysis is the one-to-one transformation of the type (22) that couples the levels. The distribution function $\phi_{i}$ then provides only the information that cannot be described by the distribution functions $\phi_{j}$ for $j<i$.

The coupling of different levels of description introduced originally in Green and Jancovici (1966) in equilibrium statistical mechanics has been extended to the dynamics in Section 2 in Grmela (2001) to write for instance the grand canonical version of the BBGKY hierarchy or the governing equations of particle mechanics coupled to fluid mechanics that are needed in direct numerical simulationssee also Grmela (2010a) - and later to write for example kinematics of complex fluids and complex solids (Grmela 2013).

Before leaving the investigation of kinematics, we note that the fact that the kinematics of the state variables (19) has appeared to be rigorously separated from the kinematics of other microscopic state variables is an argument supporting their choice.

\section{Dissipation potential $\Xi$}

In order to be able to continue the rigorous bottom-up analysis toward the irreversible and dissipative part of the time evolution, we have to investigate not only the vector fields (i.e., the time evolution equations) but also the trajectories that they generate (i.e., we have to investigate also solutions to the time evolution equations). From the trajectories, we have to then extract information that is pertinent to the chosen level of description. We shall not follow this route. Instead, we shall let ourselves be guided by nonequilibrium thermodynamics, in particular by the structure expressed in the second term on the right-hand side of Eq. 16. At the end, we shall be able, at least in some particular situations, to relate our phenomenological analysis to the 
microhydrodynamic analysis of the motion of fibers suspended in a fluid.

Following the classical nonequilibrium thermodynamics (see, e.g., De Groot and Mazur 1984), we consider the dissipation, and thus also the approach to equilibrium states, to be driven by thermodynamic forces (we shall use the symbol $\mathcal{X}$ and $\mathcal{Y}$ to denote them). At equilibrium, the thermodynamic forces disappear. We propose the following six dissipative thermodynamic forces:

$$
\begin{aligned}
& \mathcal{X}_{i}^{(1)}=\Phi_{U_{i}} \\
& \mathcal{X}_{i}^{(2)}=R_{k} D_{i k} \\
& \mathcal{Y}_{i}^{(1)}=\Phi_{W_{i}} \\
& \mathcal{Y}_{i}^{(2)}=R^{2} \mathcal{D}_{j j i} \\
& \mathcal{Y}_{i}^{(3)}=R_{j} R_{k} \mathcal{D}_{i j k} \\
& \mathcal{Y}_{i}^{(4)}=R_{i} R_{k} \mathcal{D}_{j j k}
\end{aligned}
$$

where

$$
D_{i j}=\frac{1}{2}\left(\frac{\partial \Phi_{u_{i}}}{\partial r_{j}}+\frac{\partial \Phi_{u_{j}}}{\partial r_{i}}\right)
$$

and

$\mathcal{D}_{i j k}=\frac{1}{3}\left(\frac{\partial D_{j k}}{\partial r_{i}}+\frac{\partial D_{i k}}{\partial r_{j}}+\frac{\partial D_{i j}}{\partial r_{k}}\right)$.

We note that all six of them are vectors and that they all have the physical dimension of velocity. From the physical point of view, the force $\mathcal{X}^{(1)}$ represents the friction in the relative motion (in the $\boldsymbol{R}$-space) of the particles and $\mathcal{Y}^{(1)}$, the friction in the motion of the particles in the $\boldsymbol{r}$-space. The force $\mathcal{X}^{(2)}$ is the force bringing about the hydrodynamic interactions, and the forces $\mathcal{Y}^{(2)}, \mathcal{Y}^{(3)}$, and $\mathcal{Y}^{(4)}$ are the Faxén-type forces (Lhuillier 2009) bringing about the across-the-streamline migration of suspended particles.

In order to be able to write down the second term on the right-hand side of Eq. 17, we shall now combine the thermodynamic forces (39) into the following dissipation potential:

$$
\begin{aligned}
\Xi=\int d \boldsymbol{r} \int & d \boldsymbol{R}\left(\mathcal{X}^{(1)}, \mathcal{X}^{(2)}, \mathcal{Y}^{(1)}, \mathcal{Y}^{(2)}, \mathcal{Y}^{(3)}, \mathcal{Y}^{(4)}\right) \\
& \times \frac{1}{2} g \boldsymbol{\Gamma}\left(\begin{array}{l}
\mathcal{X}^{(1)} \\
\mathcal{X}^{(2)} \\
\mathcal{Y}^{(1)} \\
\mathcal{Y}^{(2)} \\
\mathcal{Y}^{(3)} \\
\mathcal{Y}^{(4)}
\end{array}\right)
\end{aligned}
$$

where

$$
\boldsymbol{\Gamma}=\left(\begin{array}{cccccc}
\hat{\Lambda}^{(1)} & \hat{\lambda} & 0 & 0 & 0 & 0 \\
\hat{\lambda} & \hat{\Lambda}^{(2)} & 0 & 0 & 0 & 0 \\
0 & 0 & \hat{\kappa}_{11} & \hat{\kappa}_{12} & \hat{\kappa}_{13} & \hat{\kappa}_{14} \\
0 & 0 & \hat{\kappa}_{21} & \hat{\kappa}_{22} & 0 & 0 \\
0 & 0 & \hat{\kappa}_{31} & 0 & \hat{\kappa}_{33} & 0 \\
0 & 0 & \hat{\kappa}_{41} & 0 & 0 & \hat{\kappa}_{44}
\end{array}\right) .
$$

The symbols $\hat{\Lambda}, \hat{\lambda}$, and $\hat{\kappa}$ denote $3 \times 3$ matrices called material parameters. The intrinsic consistency of the final time evolution (17) restricts these matrices only by requiring that the large matrix $\boldsymbol{\Gamma}$ is positive definite. We shall postpone their specification to the later stage of the analysis. It would, of course, be possible to propose a more general $\boldsymbol{\Gamma}$ (for example, involving coupling between $\mathcal{X}$ and $\mathcal{Y}$ forces), but at present, we do not have any microhydrodynamic argument that would support such generalization. In fact, we do have microhydrodynamic arguments only for the $\mathcal{X}$ forces and for the choice of $\hat{\Lambda}^{(1)}, \hat{\Lambda}^{(2)}$, and $\hat{\lambda}$ (see Zmievski et al. $2005)$ but we do not have any additional arguments to support the choice of the forces $\mathcal{Y}$ and the matrices $\boldsymbol{\kappa}$ than those advanced in Lhuillier (2009).

By inserting (42) into (17), we can now write explicitly the second term on the right-hand side, and thus, if we combine it with the first term (26), we arrive at the complete set of the time evolution equations.

\section{Reduction from the state variables (19) to the state variables (18)}

Next, we shall pass to a reduced description by making assumptions about solutions of these equations. We assume that the material parameters entering $\Gamma$, and also the still unspecified free energy $\Phi$, are such that both $\boldsymbol{U}$ and $\boldsymbol{W}$ relax to their equilibrium values (that are $\boldsymbol{U}=\boldsymbol{W}=0$ ), much more rapidly than the rest of the state variables. We shall consider now the pseudo-equilibrium state at which $\frac{\partial \boldsymbol{U}}{\partial t}=\frac{\partial \boldsymbol{W}}{\partial t}=0$. If we write these two equations and keep only the leading terms (i.e., neglect all the terms quadratic and higher order in $\boldsymbol{U}$ and $\boldsymbol{W}$ ), we obtain

$$
\begin{aligned}
& -g\left(\frac{\partial \Phi_{g}}{\partial \boldsymbol{R}}+\hat{\Lambda}^{(1)} \mathcal{X}^{(1)}+\hat{\lambda} \mathcal{X}^{(2)}\right)=0 \\
& -g\left(\frac{\partial \Phi_{g}}{\partial \boldsymbol{r}}+\hat{\kappa}_{11} \mathcal{Y}^{(1)}+\hat{\kappa}_{12} \mathcal{Y}^{(2)}+\hat{\kappa}_{13} \mathcal{Y}^{(3)}+\hat{\kappa}_{14} \mathcal{Y}^{(4)}\right)=0 .
\end{aligned}
$$

If these equations are inserted into the third equation in Eq. 26, we obtain the kinetic Eq. 1 with

$$
\begin{aligned}
& \mathcal{X}=\mathcal{X}^{(2)} ; \mathcal{Y}^{(\mathrm{I})}=\mathcal{Y}^{(2)} ; \mathcal{Y}^{(\mathrm{II})}=\mathcal{Y}^{(3)} ; \mathcal{Y}^{(\mathrm{III})}=\mathcal{Y}^{(4)} \\
& \boldsymbol{\Lambda}=\left(\hat{\Lambda}^{(1)}\right)^{-1} ; \lambda=\left(\hat{\Lambda}^{(1)}\right)^{-1} \hat{\lambda}^{\prime} ; \\
& \boldsymbol{\kappa}=\left(\hat{\kappa}_{11}\right)^{-1} ; \boldsymbol{\kappa}^{(\mathrm{I})}=\left(\hat{\kappa}_{11}\right)^{-1} \hat{\kappa}_{12} ; \boldsymbol{\kappa}^{(\mathrm{II})}=\left(\hat{\kappa}_{11}\right)^{-1} \hat{\kappa}_{13} ; \\
& \boldsymbol{\kappa}^{(\mathrm{III})}=\left(\hat{\kappa}_{11}\right)^{-1} \hat{\kappa}_{14} .
\end{aligned}
$$


This concludes the derivation of the extended Smoluchowski -typed kinetic Eq. 1.

Stress tensor and material parameters

It remains to derive the expression (3) for the stress tensor $\sigma$ and to explain the choices (5) and (8) of the material parameters $\boldsymbol{\Lambda}, \boldsymbol{\kappa}, \boldsymbol{\kappa}^{(\mathrm{II})}, \boldsymbol{\kappa}^{(\mathrm{II})}$, and $\boldsymbol{\kappa}^{(\mathrm{III})}$.

We begin with the stress tensor $\boldsymbol{\sigma}$. Expression (28) is not, of course, anymore applicable since we have reduced the full set of equations governing the time evolution of $g, \boldsymbol{U}, \boldsymbol{W}$ just to one equation (1) which governs the time evolution of $g$. The argument of the compatibility with thermodynamics, namely the requirement that the free energy $\Phi$ remains unchanged in the externally unforced reversible time evolution (see Grmela 1985, 2008; Grmela et al. 2011), is still however applicable. The expression (3) arises from the equation

$$
\begin{aligned}
\left(\frac{d \Phi}{d t}\right)_{\mathrm{rev}}= & \int d \boldsymbol{r}\left[\Phi_{\rho}\left(\frac{\partial u_{i}}{\partial t}\right)_{\mathrm{rev}}+\Phi_{u_{i}}\left(\frac{\partial u_{i}}{\partial t}\right)_{\mathrm{rev}}\right. \\
& \left.+\int d \boldsymbol{R} \Phi_{g}\left(\frac{\partial g}{\partial t}\right)_{\mathrm{rev}}\right] \\
= & \int d \boldsymbol{r} \frac{\partial \Phi_{u_{i}}}{\partial r_{j}}\left[\sigma_{i j}+(\bullet \bullet \bullet)\right]=0 .
\end{aligned}
$$

Integration by parts (with the boundary conditions guaranteeing disappearance of the integrals over the boundaries) is made in the step leading to the second equality. The expression (3) is the equation $\left[\sigma_{i j}+(\bullet \bullet \bullet)\right]=0$. Note that the reversible part of the time evolution of $g$ is governed by the kinetic equation (1) with the last two terms on its right-hand side missing.

We now turn to the material parameters. Microhydrodynamic analysis of hydrodynamic interactions has been carried in Batchelor and Green (1972) and Batchelor (1977) (see also the other references cited in Zmievski et al. 2005 ) to the Smoluchowski-type equation. (1) without the terms involving the matrices $\boldsymbol{\kappa}, \boldsymbol{\kappa}^{(\mathrm{I})}, \boldsymbol{\kappa}^{(\mathrm{II})}$, and $\boldsymbol{\kappa}^{(\mathrm{III})}$. The material parameters $\boldsymbol{\Lambda}, \boldsymbol{\lambda}$ have thus a clear microhydrodynamic interpretation. The microhydrodynamic derivation of the Smoluchowski-type kinetic equation provides us also with the estimate of its domain of validity (semidilute suspensions).

The Faxén-type forces $\mathcal{Y}$ and the parameters $\boldsymbol{\kappa}$ have been introduced in Lhuillier (2009) phenomenologically with a very limited microhydrodynamic background. In this paper, we remain, as far as $\mathcal{Y}$ and $\boldsymbol{\kappa}$ are concerned, on the same phenomenological level. Our goal is only to incorporate $\mathcal{Y}$ and $\boldsymbol{\kappa}$ into the full context of morphology and rheology of suspensions. The choice of $\boldsymbol{\kappa}$ made in Eq. 8 is completely phenomenological, directed mainly by the requirement of simplicity. Our results (numerical solutions of the governing equations), seen as a mapping $\boldsymbol{\kappa} \rightarrow$ morphology \& rheology, provide an indirect insight into the physical meaning and the significance of the coefficients $\boldsymbol{\kappa}$.

\section{References}

Ammar A, Mokdad B, Chinesta F, Keunings R (2006) A new family of solvers for some classes of multidimensional partial differential equations encountered in kinetic theory modeling of complex fluids. J Non-Newtonian Fluid Mech 139(3):153-176

Ammar A, Mokdad B, Chinesta F, Keunings R (2007) A new family of solvers for some classes of multidimensional partial differential equations encountered in kinetic theory modelling of complex fluids: part II: transient simulation using space-time separated representations. J Non-Newtonian Fluid Mech 144(2):98-121

Ammar A, Pruliere E, Chinesta F, Laso M (2009) Reduced numerical modeling of flows involving liquid-crystalline polymers. J NonNewtonian Fluid Mech 160(2):140-156

Arnold VI (1989) Mathematical methods of classical mechanics. Graduate texts in mathematics, vol 60. Springer, New York

Batchelor GK (1977) The effect of Brownian motion on the bulk stress in a suspension of spherical particles. J Fluid Mech 83(1):97-117

Batchelor GK, Green JT (1972) The hydrodynamic interaction of two small freely-moving spheres in a linear flow field. J Fluid Mech 56(2):375-400

Beris AN, Edwards BJ (1994) Thermodynamics of flowing systems: with internal structure. Oxford engineering science series, vol 36. Oxford University Press, New York

Brady JF, Morris JF (1997) Microstructure of strongly sheared suspensions and its impact on rheology and diffusion. J Fluid Mech 348:103-139

Chinesta F, Amar A, Cueto E (2010) Recent advances and new challenges in the use of the proper generalized decomposition for solving multidimensional models. Arch Comput Methods Eng 17(4):327-350

Chinesta F, Ammar A, Leygue A, Keunings R (2011) An overview of the proper generalized decomposition with applications in computational rheology. J Non-Newtonian Fluid Mech 166(11):578592

Clebsch A (1859) Über die integration der hydrodynamischen gleichungen. J Reine Angew Math 56:1-10

De Groot SR, Mazur P (1984) Non equilibrium thermodynamics. Dover, New York

Dzyaloshinskii IE, Volovick GE (1980) Poisson brackets in condensed matter physics. Ann Phys 125(1):67-97

Euler L (1755) Principes généraux du mouvement des fluides. Mém Acad R Sci B-Lett Berl 11. See also English translation in Physica D 237:1852-1854 (2008)

Frank M, Anderson D, Weeks ER, Morris JF (2003) Particle migration in pressure-driven flow of a Brownian suspension. J Fluid Mech 493(1):363-378

Friedrichs KO (1978) Conservation equations and the laws of motion in classical physics. Commun Pure Appl Math 31(1):123-131

Friedrichs KO, Lax PD (1971) Systems of conservation equations with a convex extension. Proc Natl Acad Sci 68(8):1686-1688

Godunov SK (1961) An interesting class of quasilinear systems. Dokl. Acad. Nauk SSSR 139:520-523; English trans., Sov Math 2:947949

Godunov SK, Romenskii EI (2003) Elements of continuum mechanics and conservation laws. Kluwer Academic/Plenum Publishers, New York

Green MS, Jancovici B (1966) Cargèse lectures in theoretical physics. Gordon and Breach, New York 
Grmela M (1984a) Particle and bracket formulations of kinetic equations. Contemp Math 28:125-132

Grmela M (1984b) Bracket formulation of dissipative fluid mechanics equations. Phys Lett A 102(8):355-358

Grmela M (1985) Stress tensor in generalized hydrodynamics. Phys Lett A 111(1):41-44

Grmela M (2001) Complex fluids subjected to external influences. J Non-Newtonian Fluid Mech 96(1):221-254

Grmela M (2008) Stress tensor in fiber suspensions. Phys Lett A 372(23):4267-4270

Grmela M (2010a) Multiscale equilibrium and nonequilibrium thermodynamics in chemical engineering. Adv Chem Eng 39:75129

Grmela M (2010b) Hamiltonian and thermodynamic modeling of quantum turbulence. J Stat Phys 141(2):318-341

Grmela M (2012a) Role of thermodynamics in multiscale physics. Comput Math Appl. doi:10.1016/j.camwa.2012.11.019

Grmela M (2012b) Fluctuations in extended mass-action-law dynamics. Phys D: Nonlinear Phenom 241(10):976-986

Grmela M (2013) Extensions of nondissipative continuum mechanics toward complex fluids and complex solids. Contin Mech Thermodyn 25(1):55-75

Grmela M, Öttinger HC (1997) Dynamics and thermodynamics of complex fluids. I. Development of a general formalism. Phys Rev E 56(6):6620-6633

Grmela M, Ammar A, Chinesta F (2011) Extra stress tensor in fiber suspensions: mechanics and thermodynamics. J Rheol 55:17-42

Grmela M, Ammar A, Chinesta F (2012) One and two-fiber orientation kinetic theories of fiber suspensions. J Non-Newtonian Fluid Mech. doi:10.1016/j.jnnfm.2012.10.009

Kaufman AN (1984) Dissipative hamiltonian systems: a unifying principle. Phys Lett A 100(8):419-422
Klika V, Grmela M (2013) Coupling between chemical kinetics and mechanics that is both nonlinear and compatible with thermodynamics. Phys Rev E Stat Nonlinear Soft Matter Phys 87(1-1):012141-012141

Kröger M, Hütter M (2010) Automated symbolic calculations in nonequilibrium thermodynamics. Comput Phys Commun 181(12):2149-2157

Lhuillier D (2009) Migration of rigid particles in non-Brownian viscous suspensions. Phys Fluids 21:023302

Maîtrejean G, Ammar A, Chinesta F, Grmela M (2012) Deterministic solution of the kinetic theory model of colloidal suspensions of structureless particles. Rheol Acta 51(6):527-543

Marsden JE, Ratiu TS (1999) Introduction to mechanics and symmetry: a basic exposition of classical mechanical systems, vol 17. Springer, New York

Morrison PJ (1984) Bracket formulation for irreversible classical fields. Phys Lett A 100(8):423-427

Nott PR, Guazzelli E, Pouliquen O (2011) The suspension balance model revisited. Phys Fluids 23:043304

Öttinger HC (2005) Beyond equilibrium thermodynamics. Wiley, Hoboken

Öttinger HC (2010) Nonlinear thermodynamic quantum master equation. Properties and examples. Phys Rev A 82(5):052119

Öttinger HC, Grmela M (1997) Dynamics and thermodynamics of complex fluids. II. Illustrations of a general formalism. Phys Rev E 56(6):6633-6650

Semwogerere D, Morris JF, Weeks ER (2007) Development of particle migration in pressure-driven flow of a Brownian suspension. $\mathrm{J}$ Fluid Mech 581:437

Zmievski V, Grmela M, Bousmina M, Dagréou S (2005) Nonlinear microstructure and rheology of semidilute colloidal suspensions of structureless particles. Phys Rev E 71(5):051503 